\title{
REVIEW
}

\section{Diffusion-driven currents in organic-semiconductor diodes}

\author{
Gert-Jan AH Wetzelaer ${ }^{1}$ and Paul WM Blom ${ }^{2}$
}

Organic light-emitting and photovoltaic diodes are attractive optoelectronic devices with organic semiconductors as the main component. Whereas the transport properties in the drift regime of such diodes have been extensively investigated, the diffusion-dominated regime has only lately attracted considerable attention. In this review, we discuss diffusion-driven currents in several types of organic diodes. It is demonstrated that the ideality factor of diffusion-driven currents can be regarded as an effective tool for studying the recombination mechanisms in organic light-emitting diodes (OLEDs) and solar cells. In doublecarrier devices, such as OLEDs, the ideality factor of the current has a temperature-independent value of 2 , which indicates that nonradiative trap-assisted recombination dominates the current. By contrast, the ideality factor of the light output approaches unity, indicating that luminescence is governed by bimolecular recombination. In a single-carrier device, in which recombination is absent, the ideality factor may deviate from unity because of a small number of deeply trapped carriers. Therefore, the ideality factor of a bulk-heterojunction solar cell can deviate from unity even in the absence of trap-assisted recombination. Finally, an analytical description of the diffusion current is derived that can be used to extract contact barriers and can explain slight deviations of the ideality from unity.

NPG Asia Materials (2014) 6, e110; doi:10.1038/am.2014.41; published online 4 July 2014

Keywords: charge transport; organic semiconductors; diffusion current

\section{INTRODUCTION}

Conjugated polymers and small molecules, as typically used in organic light-emitting diodes (OLEDs) or solar cells, are undoped semiconductors. When these semiconductors are sandwiched between two electrodes, a metal-insulator-metal (MIM) diode stack is formed. The current that flows through such an organic-semiconductor diode results from the drift and diffusion of charge carriers. In this review, we focus on diffusion-driven currents in several types of organicsemiconductor diodes. Studying these diffusion currents provides important information on charge transport and recombination in organic semiconductors.

To illustrate what determines the current flow in an organicsemiconductor diode, we will describe the case of a single-carrier device with an ohmic injecting contact and a non-ohmic collection contact. In general, when electrodes with different work function are used, an internal electric field will build up, resulting from a built-in voltage $V_{\mathrm{bi}}$ across the undoped semiconductor, which equals the difference in the work functions of the electrodes. For reference, we consider the case of a hole-only MIM diode with one ohmic and one non-ohmic contact, as illustrated in Figure 1a. The collecting contact at $x=L$, where $x$ denotes the position within the diode and $L$ denotes the film thickness, is separated from the valence band by a barrier height, $\varphi_{\mathrm{b}}$. The injecting contact at $x=0$ aligns with the valence band.
For both contacts, the energy barrier to the conduction band is so large that electron injection can be neglected. The $V_{\mathrm{bi}}$ is then equal to the barrier height $\varphi_{\mathrm{b}}$.

At a high positive bias on the ohmic contact, larger than the builtin voltage, the resulting electric field becomes positive, pointing towards the collecting contact. Holes are injected and the current is dominated by drift. The current becomes limited by the uncompensated charges of the injected holes, leading to a space-charge-limited current that can be analytically described by the Mott-Gurney equation $^{1}$ as follows:

$$
J=\frac{9}{8} \varepsilon \mu \frac{\left(V-V_{\mathrm{bi}}\right)^{2}}{L^{3}},
$$

where $\varepsilon$ is the permittivity of the insulator or semiconductor, $\mu$ is the charge-carrier mobility and $V$ is the applied voltage. A large advantage of such an analytical description is that from experimental data on single-carrier devices, the charge carrier mobility can directly be obtained from current density versus voltage $(J-V)$ measurements. ${ }^{2}$

As shown in Figure 1a, we now apply a small positive bias on the injecting contact. When the bias is smaller than the built-in voltage, the electric field remains negative, pointing towards the injecting contact. The drift current because of the injected holes is therefore negative. However, the gradient in the hole density leads to the

1Zernike Institute for Advanced Materials, University of Groningen, Groningen, The Netherlands and ${ }^{2}$ Department of Molecular Electronics, Max Planck Institute for Polymer Research, Mainz, Germany

Correspondence: Professor Dr Paul WM Blom, Department of Molecular Electronics, Max Planck Institute for Polymer Research, Ackermannweg 10, Mainz 55128, Germany. E-mail: blom@mpip-mainz.mpg.de

Received 18 February 2014; revised 30 April 2014; accepted 30 April 2014 

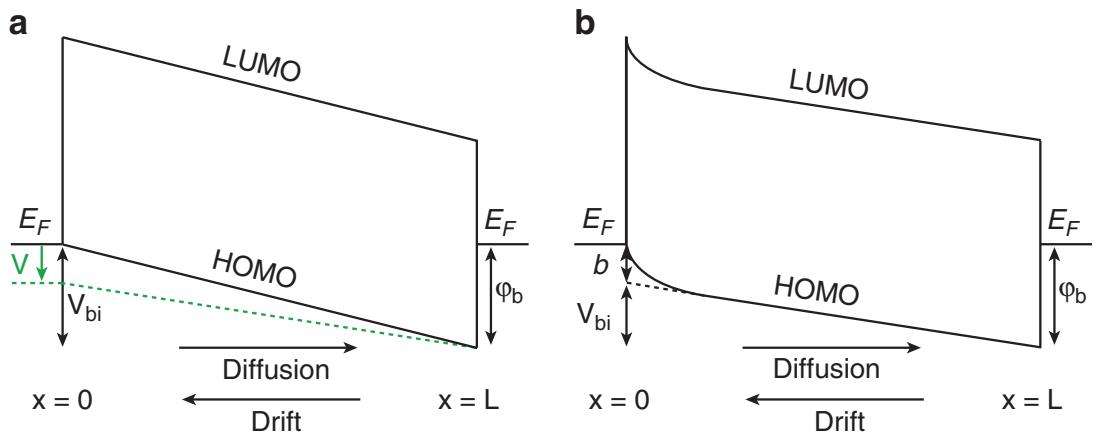

Figure 1 Energy-band diagram of an organic hole-only MIM diode. The layer thickness is $L$ and $x$ denotes the position in the diode. The contact at $x=0$ is Ohmic, whereas the collecting contact at $x=L$ is separated from the valence band by a barrier height $\varphi_{\mathrm{b}}$. (a) Thermal equilibrium and upon applying a small positive bias smaller than the built-in voltage (dashed). (b) Band diagram at thermal equilibrium when including band bending due to injected holes at the ohmic contact.

diffusion of holes towards the collecting contact. The net positive current then results from the positive diffusion current that dominates over the negative drift current. To date, the diffusion-limited current in organic MIM diodes has been analyzed using the classical Shockley diode equation, ${ }^{3}$ given as follows:

$$
J=J_{0}\left[\exp \left(\frac{q V}{\eta k T}\right)-1\right]
$$

where the saturation current density $J_{0}$ is normally taken as a fit parameter and $\eta$ is an empirical ideality factor, $k$ is the Boltzmann constant, $T$ is the temperature and $q$ is the elementary charge. For an ideal $p-n$ junction diode without trapping of charge carriers and for which recombination is absent or governed by bimolecular recombination, the ideality factor is expected to be equal to unity. For organic semiconductors, the ideality factor contains important information on the transport and recombination processes in organic semiconductors, as will be discussed in the following sections.

\section{EFFECT OF TRAP-ASSISTED RECOMBINATION ON THE IDEALITY FACTOR}

As a starting point, we will discuss OLEDs. The light-emitting organic semiconductor, which is the conjugated polymer super-yellow poly ( $p$-phenylene vinylene) in this case, is sandwiched between an ohmic hole and electron contact. Owing to the difference in the work functions of the electrodes, a $V_{\mathrm{bi}}$ across the device exists. The current density-voltage characteristic of an OLED (Figure 2) therefore exhibits three discernable regimes: at low voltages, the current is dominated by parasitical currents between the electrodes, called the leakage current, depending linearly on voltage. The second regime, with $V$ lower than $V_{\mathrm{bi}}$, is diffusion-dominated and has an exponential dependence on voltage, as given by Equation (2). The ideality factor can be regarded as a measure of the slope of the $J-V$ characteristics on a semilogarithmic plot. In the absence of recombination, the idealdiode equation should apply, where $\eta$ equals unity. At the built-in voltage, a transition from the exponential regime to the driftdominated, space-charge-limited regime occurs (regime 3), which is characterized by a quadratic dependence of the current on the voltage in the case of trap-free transport, according to Equation (1).

However, as first described by Sah et al. ${ }^{4}$, the ideality factor of a classical $p-n$ junction is affected by trap-assisted recombination in the space-charge region. Here, electrons immobilized in trapping sites recombine with free holes, as described by the Shockley-Read-Hall (SRH) formalism. ${ }^{5,6}$ When SRH recombination is the dominant loss mechanism, the ideality factor is expected to be precisely equal to $2 .^{4}$

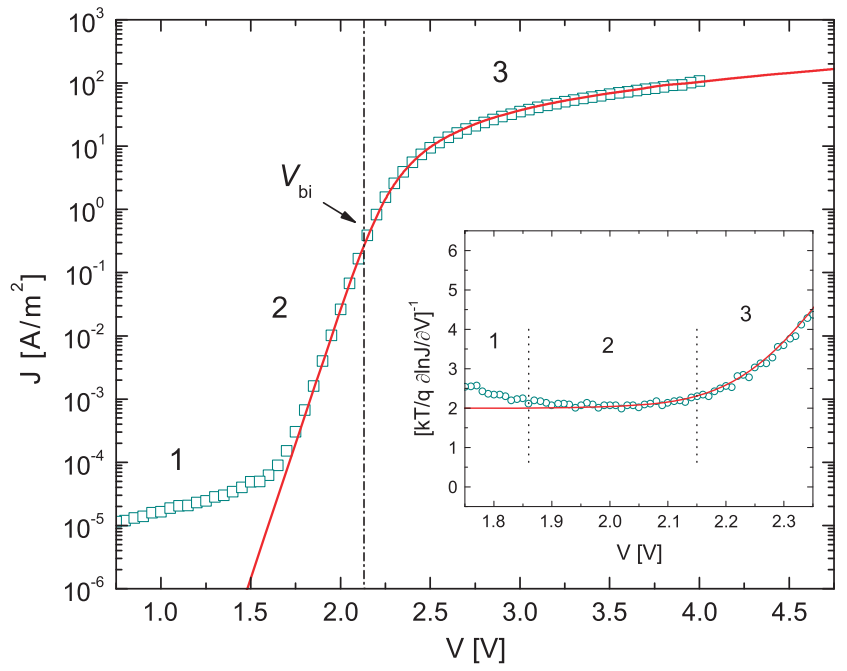

Figure 2 Experimental $J-V$ characteristics (symbols) of a 43-nm superyellow poly( $p$-phenylene vinylene) (SY) OLED and the corresponding calculations (solid line) from a drift-diffusion model, using $C_{n}=C_{p}=2 \times 10^{-18} \mathrm{~m}^{3} \mathrm{~s}^{-1}$ and $N_{t}=5 \times 10^{22} \mathrm{~m}^{-3}$, with a trap depth of $0.5 \mathrm{eV}$. The inset shows the differential plot of the data and simulation according to Equation (3). The leakage (1), diffusion (2) and drift (3) regimes are indicated.

Thus, the ideality factor can be used as a fingerprint for trap-assisted recombination.

Conventionally, the ideality factor is determined by fitting the experimental data with Equation (2). However, to prevent erroneous fitting, we determine the ideality factor by numerical differentiation according to the following equation:

$$
\eta=\left(\frac{k T}{q} \frac{\partial \ln J}{\partial V}\right)^{-1} \text {. }
$$

By plotting this quantity against voltage, as shown in the inset of Figure 2, the three regimes in the $J-V$ characteristics can be distinguished again, where the ideality factor is obtained from the plateau value. The plateau value can be regarded as the steepest exponential incline of the $J-V$ characteristics. For a correct determination of the ideality factor, it is important that the exponential part be clearly discernable, requiring low leakage currents and high current densities in the space-charge limit.

As observed in the inset of Figure 2, an ideality factor of precisely 2 was determined for the super-yellow poly( $p$-phenylene vinylene) 
device, in exact correspondence to the value that is predicted from the Sah-Noyce-Shockley theory ${ }^{4}$ for trap-assisted recombination. In addition, both the drift and diffusion regimes can be accurately described using a numerical drift-diffusion model, ${ }^{7}$ which next to Langevin recombination includes an additional SRH recombination mechanism, for which the recombination rate is given as follows:

$$
R_{\mathrm{SRH}}=C_{n} C_{p} N_{t}\left(n p-n_{1} p_{1}\right) /\left[C_{n}\left(n+n_{1}\right)+C_{p}\left(p+p_{1}\right)\right],
$$

where $C_{n}$ and $\mathrm{C}_{p}$ are the capture coefficients for electrons and holes, respectively; $N_{t}$ is the density of electron traps; $n$ and $p$ are the electron and hole densities, respectively; and $n_{1} p_{1}=n_{i}^{2}$ is their product under equilibrium conditions if the Fermi level coincides with the position of the recombination centers, where $n_{i}$ denotes the intrinsic carrier concentration in the sample. An ideality factor of 2 in the current of an OLED indicates that the dominant recombination mechanism is trap-assisted. However, previous studies ${ }^{8,9}$ concluded that charge recombination in organic semiconductors is a bimolecular process of the Langevin type, ${ }^{10}$ controlled by the diffusion of oppositely charged free carriers towards each other in their mutual Coulomb field. The Langevin recombination rate is expressed as follows:

$$
R_{L}=\frac{q}{\varepsilon}\left(\mu_{n}+\mu\right)\left(n p-n_{i}^{2}\right),
$$

where $\varepsilon$ is the dielectric constant, and $\mu_{n}$ and $\mu_{p}$ are the electron and hole mobilities, respectively. This apparent discrepancy can be resolved by also studying the ideality factor of the luminance $(L)$ versus voltage characteristics, analogous to the case for the current density. When plotting the ideality factors of the current and luminance (Figure 3), it appears that a distinct dissimilarity in the slope of the exponential part of the characteristics is present, which is observed for both super-yellow poly( $p$-phenylene vinylene) and the phenyl-substituted poly( $p$-phenylene vinylene) derivative NRS-PPV. In contrast to the case for the current density $(\eta=2)$, the ideality factor determined from the $L-V$ characteristics approaches unity, which is consistent with Langevin recombination. This finding is a strong indication that in these diodes, nonradiative trap-assisted recombination is the most dominant and controls the current,

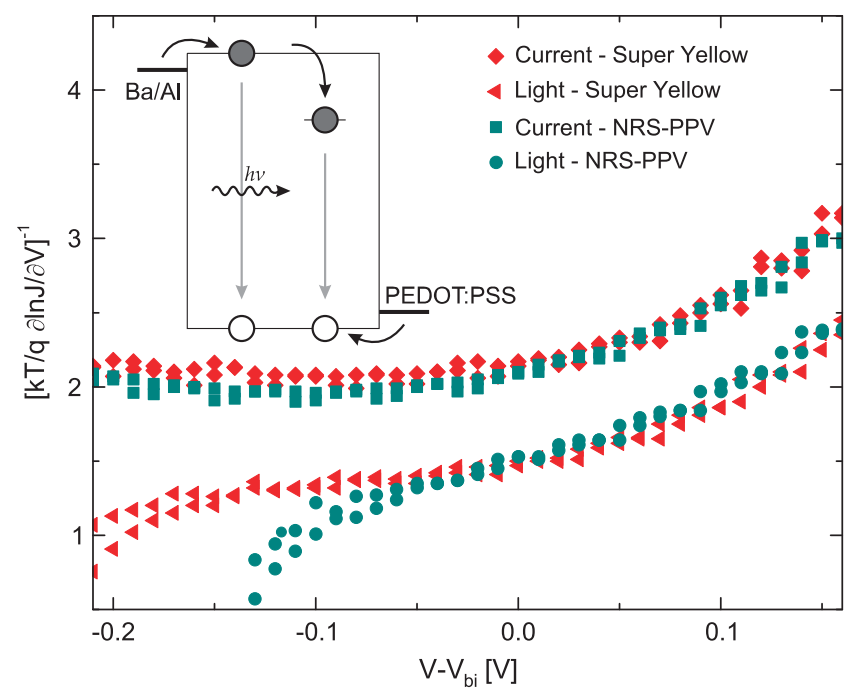

Figure $3 \eta-V$ characteristics of SY and NRS-PPV OLEDs derived from the current and the luminance. The luminance ideality factor approaching unity indicates that light emission is governed by a Langevin process. The inset shows a schematic representation of the recombination mechanisms. whereas the light emission is a result of a competing bimolecular recombination process.

To experimentally verify this hypothesis, we studied the $L-V$ characteristics of a diode in which emissive recombination from trapped charge carriers is present, as is the case in host-guest systems, frequently applied in highly efficient organic LEDs. An ideal compound for this test is a state-of-the-art white-emitting polymer (Merck), ${ }^{11}$ in which green- and red-emitting chromophores are incorporated in the blue-emitting backbone. In this polymer, the highest occupied molecular orbital (HOMO) levels of the chromophores align with the HOMO of the blue host, whereas the lowest unoccupied molecular orbital (LUMO) levels of the chromophores are substantially below the LUMO of the blue host because of the smaller band gap. ${ }^{12}$ The chromophores are therefore expected to trap electrons. Hence, the blue part of the emission spectrum is assumed to arise from Langevin recombination, whereas the red emission can be ascribed to the recombination of holes with electrons that are trapped on the chromophore. By measuring the $L-V$ characteristics through selective optical filters, the different recombination mechanisms can be disentangled. Therefore, a blue dichroic filter (serving as a bandpass filter) and a 550-nm longpass filter were used, splitting the electroluminescence (EL) spectrum into a blue and a red component. As observed in Figure 4a, the ideality factor of the current density again amounts to 2, which is consistent with materials without chromophores. Remarkably, Figure $4 \mathrm{~b}$ reveals a clear difference in the slope of the $L-V$ characteristics when either the red or blue part of the spectrum is measured. The ideality factor for the red light also amounts to 2, confirming that the red emission originates from SRH recombination of an electron that is trapped on the chromophore with a free hole. For the blue emission, a substantially lower ideality factor approaching unity was observed, which is consistent with bimolecular Langevin recombination. The unfiltered $L-V$ characteristics are governed by the trap-assisted recombination from the red chromophores because this mechanism prevails in the low voltage regime. The observation of a luminance ideality factor shifting to 2 when emissive traps are introduced strongly substantiates the idea that the ideality factor of 2 in OLEDs originates from trap-assisted recombination.

To summarize this section, trap-assisted recombination in polymer light-emitting diodes has been identified as the dominant recombination mechanism at low bias, which results in an ideality factor of 2. The light output, however, originates from a competing bimolecular recombination process, with an ideality factor much closer to unity. Therefore, trap-assisted recombination is typically nonradiative. Trap-assisted and bimolecular recombination can be disentangled for a white-emitting polymer, in which both mechanisms are radiative.

\section{IDEALITY FACTOR AND THE EINSTEIN RELATION}

In the previous section, the effect of trap-assisted recombination on the ideality factor was discussed. However, an ideality factor above unity could also be caused by a violation of the classical Einstein relation. The Einstein relation describes the ratio between diffusivity and mobility according to the following equation ${ }^{13}$ :

$$
\frac{D}{\mu}=\frac{k T}{q}
$$

where $D$ is the diffusion coefficient and $\mu$ is the charge carrier mobility. However, the validity of this relation in disordered materials, such as organic semiconductors, is nontrivial. As charge transport in these disordered semiconducting systems is generally characterized by 

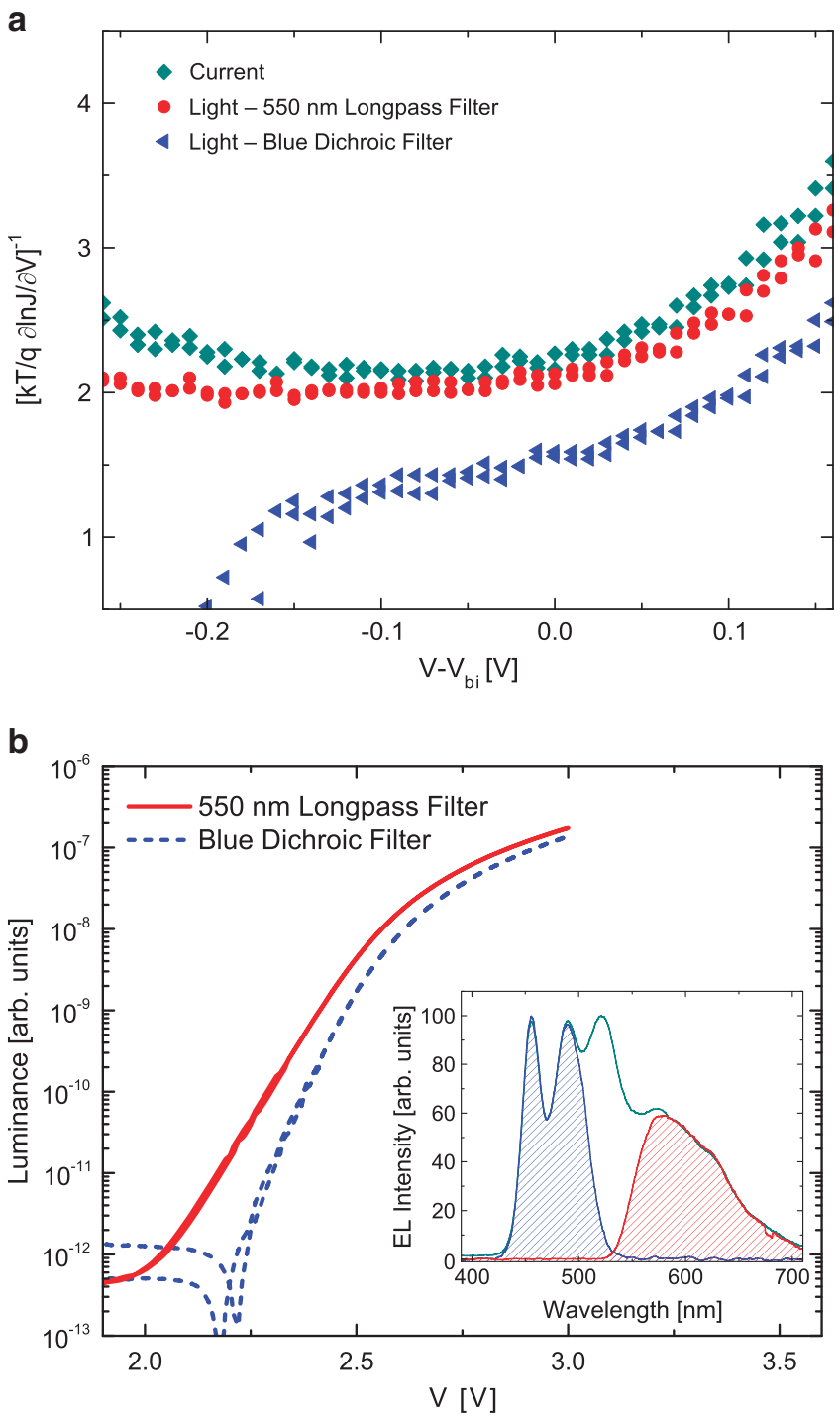

Figure $4 \eta-V($ a) and $L-V$ (b) characteristics of a 30-nm white OLED using selective optical filters, indicating the difference in ideality factor for the red- and blue-light components. The inset shows the corresponding EL spectrum, where the filled areas represent the spectra of the filtered light.

a hopping process between localized sites in a Gaussian or exponential density-of-states (DOS) distribution, ${ }^{14-16}$ their physical properties deviate significantly from classical semiconductor models. Therefore, the applicability of the Einstein relation to disordered semiconductors has been a matter of intensive research, where it is important to distinguish between equilibrium and nonequilibrium effects. Because of large disorder, the time for carriers to equilibrate can be very large, such that nonequilibrium transport is not uncommon in disordered semiconductors. Numerous theoretical and experimental studies ${ }^{17-20}$ concluded that the Einstein relation is violated under nonequilibrium conditions, mainly because of the electric field dependence of the diffusivity being larger than the field response of the mobility. However, even under quasiequilibrium conditions, it has been proposed that Equation (5) does not hold for disordered semiconductors. $^{21-23}$ This has been attributed to their non-classical DOS distributions, requiring the application of an Einstein relation generalized for an arbitrary energy-distribution function of charge density and localized states. The DOS of organic semiconductors is generally described by a Gaussian energy distribution, for which the generalized Einstein relation is expressed as follows ${ }^{22}$ :

$$
\frac{D}{\mu}=\frac{p}{q \frac{\partial p}{\partial E_{\mathrm{f}}}}=g(p, T) \frac{k T}{q},
$$

where $p$ is the charge carrier density and $E_{\mathrm{f}}$ is the quasi-Fermi level. The factor $g(p, T)$ can be regarded as a dimensionless enhancement function of the classical Einstein relation that follows from the DOS variance $\sigma$. Thus, the Einstein relation becomes charge-density- and temperature-dependent, with $g$ increasing for increasing density and decreasing temperature. However, even though such a derivation is commonly accepted, Baranovskii et al. ${ }^{19}$ noted that Equation (6) is only valid when $\mu$ and $D$ are considered independent of energy, which is not the case for hopping transport or relaxation in an exponential band tail. In a later publication, ${ }^{24}$ these authors concluded that the classical Einstein relation holds in thermal equilibrium, based on computer simulations. Moreover, direct experimental evidence for the applicability of the generalized Einstein relation is poor. Relatively large deviations from the classical Einstein relation have been reported in an experimental study ${ }^{25}$ based on measuring the density and its change with Fermi-level position in a field-effect transistor using Kelvin probe force microscopy. As implied by the generalized Einstein relation in Equation (6), these measurements would yield the diffusivity-mobility ratio. One of the most direct methods to probe the Einstein relation in organic semiconductors is by measuring the current density-voltage characteristics of homojunction diodes in the diffusion-dominated regime, as demonstrated by Harada et al. ${ }^{23}$ The (generalized) Einstein relation was shown to be directly represented in the common Shockley diode equation, which describes the $J-V$ characteristics, given as follows ${ }^{3}$ :

$$
J=J_{0}\left[\exp \left(\frac{q V}{g k T}\right)-1\right]
$$

where $J_{0}$ is the saturation current density and $g$ is the enhancement factor of the classical Einstein relation (Equation (6)). Note that the general diode equation is usually expressed as in Equation (2), with $\eta$ representing the ideality factor. The ideality factor can thus be regarded as being equivalent to the enhancement factor $g$ in Equation (6) and equals unity when the classical Einstein relation applies, under the condition that recombination is absent. Note that a carrier density dependence of the mobility could also affect the ideality factor. When the mobility increases with density-and therefore also with voltage-in the exponential diffusion-dominated regime, the $J-V$ characteristics will become steeper, affecting the ideality factor. Although the mobility in disordered organic semiconductors is indeed dependent on carrier density, the mobility is observed to be relatively constant in the typical voltage and density range in which the ideality factor is measured. ${ }^{26}$ Such mobilitydensity behavior is characteristic of a Gaussian DOS distribution rather than an exponential DOS distribution. Indeed, theoretical analysis has demonstrated that for a Gaussian DOS distribution, the ideality factor and its temperature dependence are almost equal to the Einstein relation enhancement. ${ }^{27,28}$

Consistent with other studies, Harada et al. ${ }^{29,30}$ measured a temperature-dependent ideality factor, in accordance with the $g \propto 1 / T$ dependence predicted by the generalized Einstein relation. ${ }^{22,27}$ However, the devices studied by Harada et al. were double-carrier $\mathrm{p}-\mathrm{i}-\mathrm{n}$ junctions, for which the ideality factors possibly are affected by the presence of trap-assisted recombination. To study the ideality factor in the absence of recombination, we prepared organic singlecarrier diodes, where one electrode ensures ohmic charge injection, 
whereas the counter electrode blocks the injection of charges of opposite sign. Therefore, the current is due to one type of carrier only, and recombination is absent.

Remarkably, the temperature-resolved $\eta-V$ characteristics of a $[6,6]$-phenyl- $\mathrm{C}_{61}$-butyric acid methyl ester (PCBM) electron-only diode in the inset in Figure 5 show that the ideality factor, although slightly deviating from unity, is invariant over the entire temperature range. Such temperature-independent characteristics were observed for a range of organic semiconductors. ${ }^{31}$ The observation of a temperature-independent ideality factor clearly contradicts the generalized Einstein relation, for which the disorder-induced enhancement scales inversely with temperature. In previous studies, ${ }^{23,29,30}$ ideality factors were observed to increase with decreasing temperature. We found that this apparent increase in the ideality factor is an experimental artifact that is caused by a too large leakage current (regime 1 in the $J-V$ characteristics in Figure 5). In that case, the leakage current masks the diffusion regime as the drift current decreases upon cooling due to the temperature dependence of the mobility, ${ }^{32}$ and the plateau in the $\eta-V$ plot can no longer be discerned. ${ }^{33}$ The apparent ideality factor will then be too high and temperature-dependent. Therefore, a reliable measurement of the ideality factor requires low leakage currents and high drift currents, enabling the observation of a clearly discernable exponential diffusion regime, as is the case for the measurement depicted in Figure 5.

Another interesting feature appears when comparing the ideality factors of a range of organic semiconductors as a function of their room temperature carrier mobility, as depicted in Figure 6. The ideality factor was determined from the temperature-independent plateau value of the differentiated characteristics, and the room temperature zero-field mobility was determined by fitting the $J-V$ characteristics in the drift regime with the well-known Mott-Gurney square law (Equation (1)). ${ }^{1}$ Craciun et al. ${ }^{32}$ have demonstrated that there is a universal relation between the mobility and its temperature activation. It is well established that the temperature dependence is a direct measure of the intrinsic disorder of the organic semiconductor. ${ }^{14,15}$ Thus, the mobility can be regarded as a measure for the width of the DOS distribution. From Figure 6, it

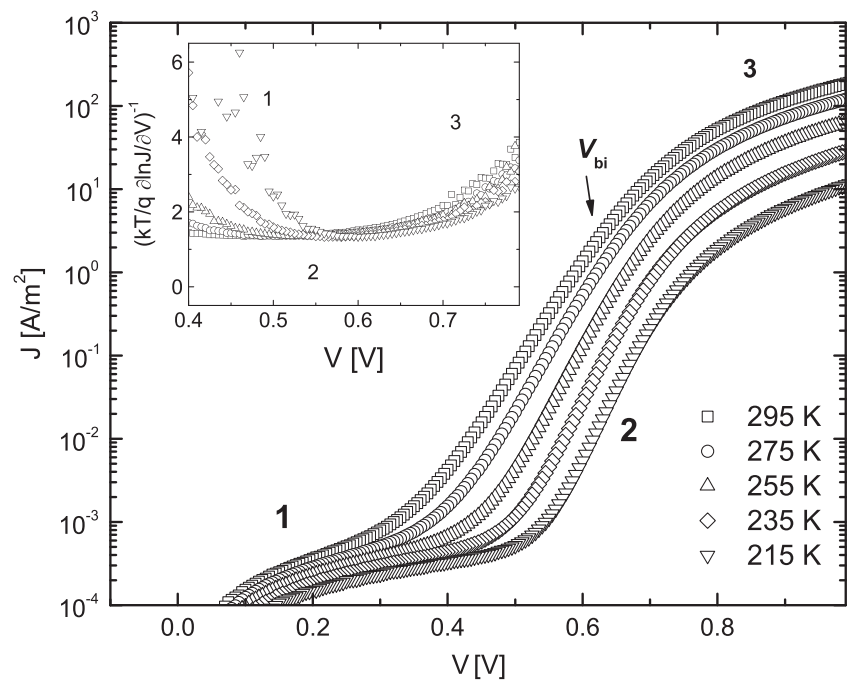

Figure 5 Temperature-dependent $J-V$ characteristics of a PCBM electrononly diode. The inset shows the differential plot of the data according to Equation (3). The leakage (1), diffusion (2) and drift (3) regimes are indicated. appears that the value of the ideality factor is not related to intrinsic disorder of the semiconductor. In addition to the observed temperature independence, this finding again contradicts the generalized Einstein relation derived for a Gaussian DOS. The temperature independence of the ideality factor would be consistent with the classical Einstein relation. However, the deviation from unity indicates that the Einstein relation is disturbed. Nenashev et al. ${ }^{20}$ recently demonstrated that the Einstein relation in disordered threedimensional hopping systems is violated even at small electric fields, which is mainly due to energetically deep, rare sites controlling the field dependence of the diffusivity. As the density of these sites is rather low, these sites do not affect the charge carrier mobility. Because of the long escape time from these deep states, deeply trapped carriers are not in thermal equilibrium with free carriers in the transport sites.

From this section, it can be concluded that the generalized Einstein relation derived for a Gaussian DOS distribution does not accurately describe the ideality factor behavior of organic single-carrier diodes. Although the ideality factor deviates from unity, it does not depend on temperature. In addition, the ideality factor exhibits no clear relation with energetic disorder. These observations cannot be explained with a generalized Einstein relation derived for a Gaussian DOS distribution. It is proposed that a nonequilibrium situation due to a small number of deep trapping sites is responsible for the observed ideality factors.

\section{IDEALITY FACTOR OF DOUBLE-CARRIER DEVICES}

If deep trapping sites are the origin of the Einstein relation enhancement in single-carrier diodes, their effect would be obscured in double-carrier diodes, that is, OLEDs, in which deep sites within the band gap act as recombination centers. Trapped charges will be released by free carriers of the opposite sign, neutralizing their effect on the transport. In section 2, it was shown that the ideality factor of an OLED generally has a value of 2 , which originates from the presence of a dominant trap-assisted SRH recombination mechanism in the diffusion regime. ${ }^{33}$ Furthermore, free-carrier recombination of the Langevin type was observed to result in an ideality factor very close to unity; this was visualized in a white-emitting polymer,

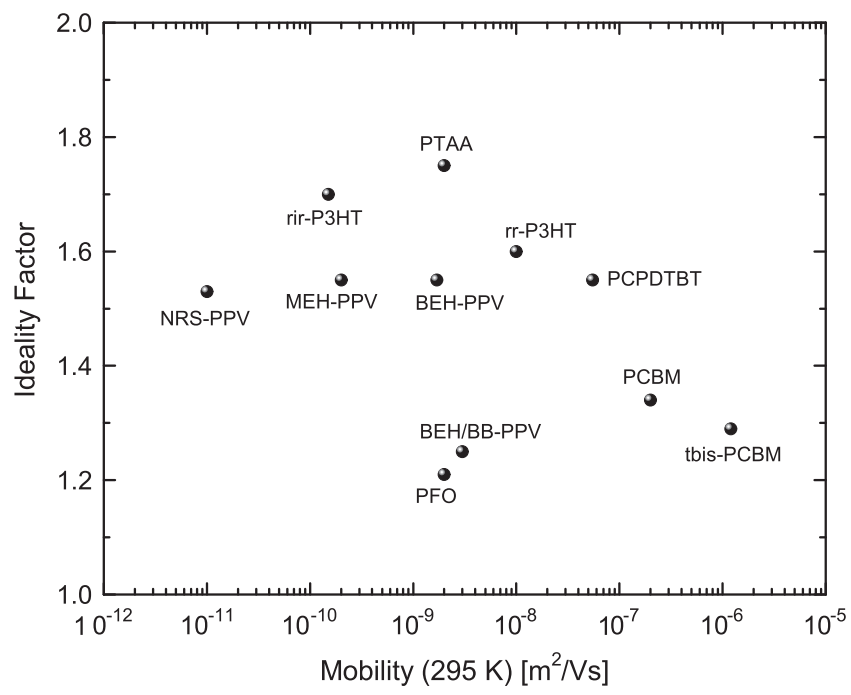

Figure 6 The ideality factor of the investigated materials as a function of their zero-field mobility at $295 \mathrm{~K}$, which can be regarded as a measure for the degree of disorder. This shows that the ideality factor and disorder are not related in these materials. 
in which red chromophores incorporated in the blue-emitting backbone function as emissive traps. Consequently, the red part of the emission arises from an SRH mechanism, whereas the blue emission is a result of Langevin recombination. The question is whether a disruption of the classical Einstein relation would still result in an SRH and Langevin ideality factor of 2 and 1 in the light emission, respectively. Therefore, device simulations were performed using a numerical drift-diffusion model, ${ }^{7}$ in which the Einstein relation could be modified arbitrarily. It was observed that the introduction of an enhancement factor $g$ simply modifies the SRH and Langevin ideality factors to $2 g$ and $1 g$, respectively. Figure 7 shows the luminance ideality factor for the red (SRH) part of the emission, which has a temperature-independent value of 2 . As a result, the data can only be described using the classical Einstein relation $(g=1)$. In addition, the $\eta-V$ characteristics of the blue (Langevin) part of the emission (inset Figure 7) show excellent agreement with the simulations using the classical Einstein relation, complementing the results for the red emission. This finding confirms that the classical Einstein relation is valid $(g=1)$ and that deep trap states govern the enhancement in single-carrier diodes.

As a final step to confirm the validity of the classical Einstein relation, an organic solar cell has been considered. In organic solar cells, a blend of electron- and hole-transporting materials is used, for which transport of both carriers can be regarded as virtually trap-free. ${ }^{7}$ Consequently, recombination in these devices arises predominantly from free charge carriers rather than trapped charge carriers. At the open-circuit voltage $\left(V_{\mathrm{oc}}\right)$, the current is zero, implying that recombination and photogeneration of charges cancel. ${ }^{34}$ As there is no net current, a balance between drift and diffusion exists, creating an excellent environment to study the Einstein relation. The ideality factor under illumination can be determined by measuring the dependence of $V_{\mathrm{oc}}$ on the logarithm of the light intensity, which scales linearly with the photogenerated current $J_{\mathrm{ph}}$. The current density under illumination is given by

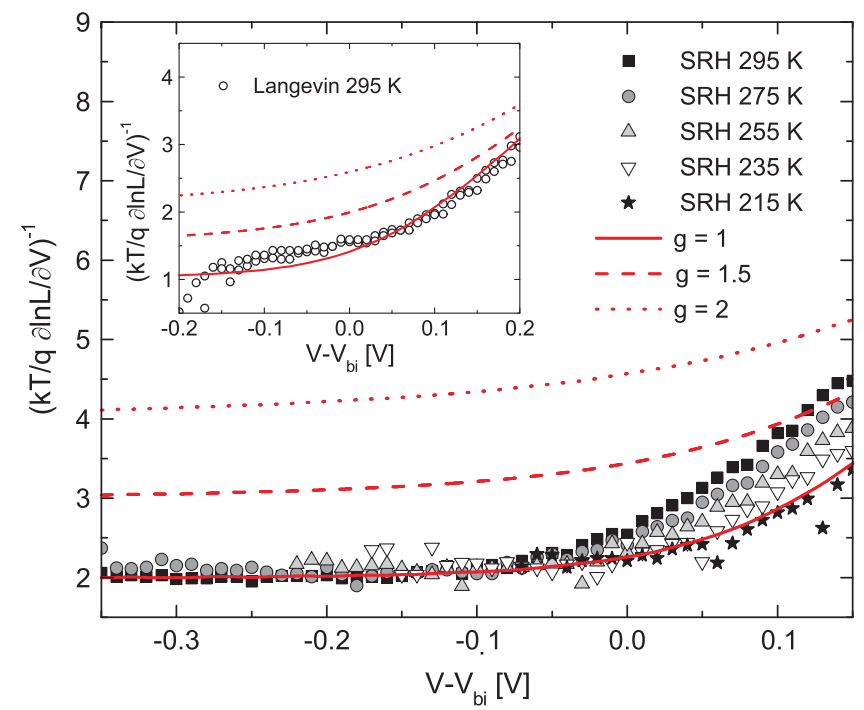

Figure 7 Ideality factor derived from the luminance $(L)$ for a $30-n m$ white OLED at different temperatures. A 550-nm longpass filter was used to selectively transmit the red emission originating from $\mathrm{SRH}$ recombination. The inset shows the luminance ideality factor using a blue bandpass filter, measuring the blue emission originating from Langevin recombination. The lines represent the simulations from a drift-diffusion model using different values for the Einstein relation enhancement $g$.
$J_{\mathrm{L}}=J_{\mathrm{D}}-J_{\mathrm{ph}}$, with $J_{\mathrm{D}}$ representing the dark-current density. Subsequently, the open-circuit voltage is given by $\left(J_{L}=0\right)$ :

$$
V_{\mathrm{oc}}=(\eta k T / q) \ln \left(J_{\mathrm{ph}} / J_{0}+1\right) .
$$

Previous research ${ }^{29}$ revealed a $k T / q$ dependence of the $V_{\text {oc }}$ on the logarithm of the incident light intensity $(I)$, which is equivalent to an ideality factor of 1 under illumination. Equivalently to the case of the dark ideality factor, the light-intensity dependence of $V_{\mathrm{oc}}$ is modified to $g k T / q$ when the Einstein relation is enhanced, as evidenced by simulations from the drift-diffusion model (Figure 8). Hence, it is immediately obvious that a $k T / q$ dependence of $V_{\text {oc }}$ versus $\ln (I)$ can only be possible when $g=1$, that is, the classical Einstein relation applies.

Although the classical Einstein relation provides a good description of the experimental data, it is not completely clear why the classical rather than the generalized Einstein relation appears to hold, especially because the generalized relation is the fundamentally expected result when a Gaussian DOS distribution is considered. In certain cases, the carrier density and energetic disorder might be too low to have a significant effect on the Einstein relation. In addition, the enhancement effect of the generalized Einstein relation stems mostly from charge carriers situated below the Fermi level. Removal of these carriers by recombination might result in the Einstein relation retaining its classical form, which is analogous to the previously described case of the removal of deeply trapped carriers. Another possible reason could be that there is a certain cutoff in the Gaussian DOS distribution, ${ }^{22}$ as a tail in the Gaussian distribution is then absent and therefore does not occupy charge carriers. In that case, the classical Einstein relation would be valid up to a higher carrier density, whereas other signs of a Gaussian DOS, such as a density-dependent mobility, are still present.

In summary, this section demonstrated that the classical Einstein relation can be used to describe the transport in double-carrier devices such as OLEDs or organic solar cells under illumination. Numerical simulations with an enhanced Einstein relation fail to describe the ideality factor data. Therefore, it appears that the classical Einstein relation is valid when deeply trapped carriers are removed via recombination.

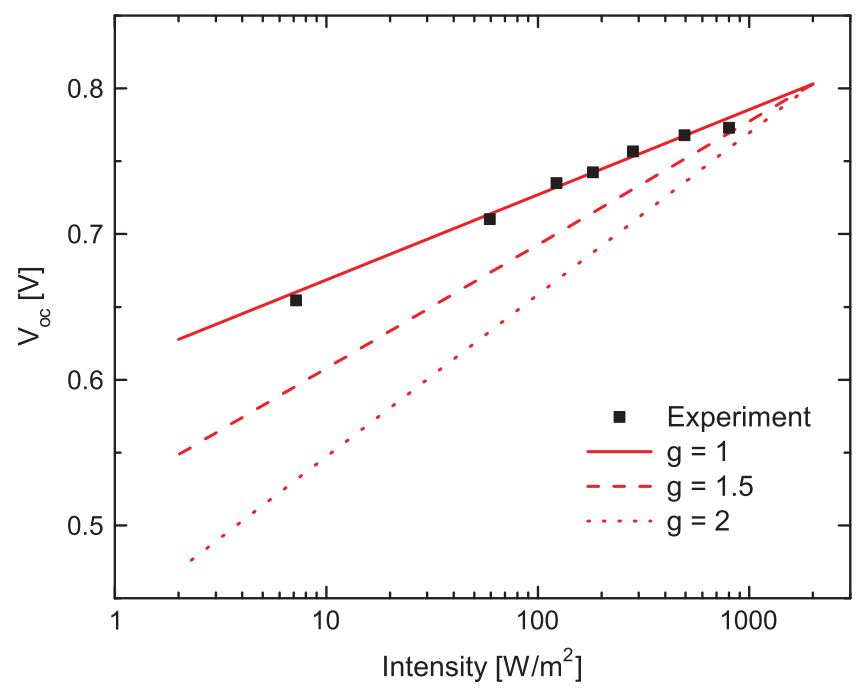

Figure $8 V_{\text {oc }}$ versus light-intensity data (symbols) of a PPV:PCBM solar cell from the study by Koster et al. ${ }^{29}$ The lines represent the simulations from a drift-diffusion model using different values for the Einstein relation enhancement $g$, normalized at $I=2000 \mathrm{~W} \mathrm{~m}^{-2}$. 


\section{IDEALITY FACTOR IN ORGANIC SOLAR CELLS}

In the previous sections, we discussed the ideality factor of OLEDs and single-carrier diodes. In principle, a bulk-heterojunction solar cell is very similar to a simple OLED. The bulk heterojunction can be described with an effective medium approach, in which the donoracceptor blend is treated as one semiconductor. ${ }^{7}$ The electron transport occurs through the LUMO of the acceptor, whereas the hole transport occurs through the HOMO of the donor.

A frequently used acceptor material is the fullerene derivative PCBM, which exhibits trap-free electron transport. ${ }^{35}$ Consequently, because both hole (donor) and electron (acceptor) traps are absent in polymer:fullerene systems, the electron-hole recombination between the donor and acceptor is expected to be bimolecular. However, recent studies reported the presence of trap-assisted recombination in polymer:fullerene bulk-heterojunction solar cells, ${ }^{36,37}$ despite the virtually trap-free electron and hole transport. These conclusions were typically based on measurements of the dark-current ideality factor.

As mentioned previously, the ideality factor can be determined from the slope of the exponential regime of dark $J-V$ characteristics on a semilogarithmic plot (Equation (3)) or by measuring the dependence of $V_{\mathrm{oc}}$ on the logarithm of the light intensity. Therefore, the ideality factor can be measured in an alternative way under illumination. From dark-current measurements on polymer:fullerene organic solar cells, ideality factors of typically 1.3-2.0 have been reported. ${ }^{29,36-38}$ Furthermore, it was first demonstrated by Mandoc et al. ${ }^{39,40}$ that the presence of trap-assisted recombination in organic solar cells can, as implied by Equation (8), be visualized by measuring the open-circuit voltage $\left(V_{\mathrm{oc}}\right)$ of an organic solar cell as a function of the incident light intensity. In these experiments on all-polymer solar cells, ${ }^{40}$ a deviation of $\eta$ from unity was also observed due to trapassisted recombination. This result appears to be consistent with the observation of $\eta$ being larger than unity in dark-current measurements. Consequently, an ideality factor greater than unity is often used as evidence for a dominant trap-assisted recombination process in organic solar cells. ${ }^{36-38}$

However, it appears that an unresolved discrepancy exists between the dark ideality factor and the light-intensity dependence of $V_{\mathrm{oc}}$,

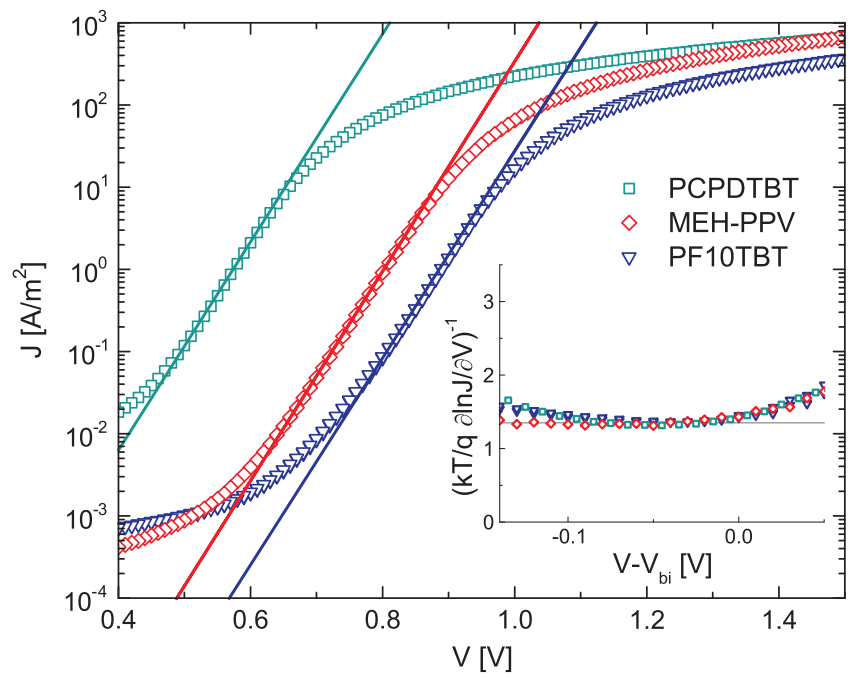

Figure 9 Dark $J-V$ characteristics of PCPDTBT:PCBM, MEH-PPV:PCBM and PF10TBT:PCBM solar cells. The solid lines are fits to the exponential regime of the characteristics. The inset shows the differential plot of the data according to Equation (3). which should have equal values according to Equations (3) and (8). A dark ideality factor larger than unity is often measured for organic solar cells of which both electron and hole transports are demonstrated to be trap-free, which is in contradiction with the appearance of trap-assisted recombination to explain the ideality factor. Furthermore, $V_{\text {oc }}$ versus light intensity measurements that also revealed a deviation from unity ${ }^{40}$ were performed on all-polymer solar cells, for which the electron transport was observed to be clearly trap-limited. However, solar cells with trap-free transport indeed do exhibit an ideality factor of unity when $V_{\mathrm{oc}}$ versus light intensity is measured, whereas in the dark current, a deviation from unity is still observed. ${ }^{29}$ Attributing the occurrence of a dark ideality factor larger than unity to trap-assisted recombination is therefore highly questionable.

In Figure 9, the dark $J-V$ characteristics for several polymer:fullerene solar cells are depicted. The characteristics exhibit typical diode behavior, with a leakage contribution at low voltages and an exponential regime at intermediate voltages, with a transition to the space-charge-limited regime at the $V_{\mathrm{bi}}$. The $V_{\mathrm{bi}}$ is determined by the work function difference between the electron- and hole-extracting electrodes. As observed in the inset in Figure 9, where the differentiated characteristics according to Equation (3) are plotted as a function of voltage, the ideality factors for all these solar cells amount to an identical value of $\sim 1.3$. By contrast, the slope $S$ of the $V_{\text {oc }}$ versus light intensity characteristics equals $k T / q$, as depicted in Figure 10. This result can be interpreted as an ideality factor of 1 under illumination, clearly indicating the dominance of bimolecular recombination. ${ }^{29}$

In the previous section, we demonstrated that the ideality factor often deviates from unity even in single-carrier diodes of a single material, where recombination is completely absent. ${ }^{31}$ This deviation from unity was ascribed to a violation of the Einstein relation, caused by a small number of deeply trapped carriers that are not in thermal equilibrium with free carriers in the transport sites. It was demonstrated that by releasing these trapped carriers by recombination, quasiequilibrium is recovered such that the Einstein relation is valid again. As the occurrence of a small number of deeply trapped carriers can be material-dependent, each component of the

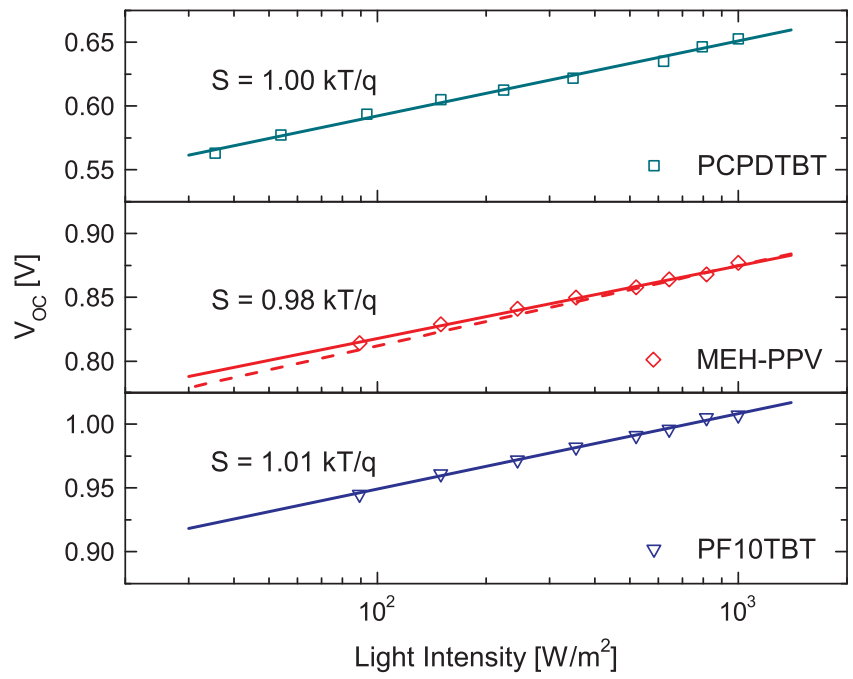

Figure $10 V_{o c}$ versus incident light intensity for PCPDTBT:PCBM, MEHPPV:PCBM and PF10TBT:PCBM solar cells. The solid lines represent the best fits to the data, for which the slope $S$ is indicated. The dashed line represents the slope $(S=1.07 \mathrm{kT} / \mathrm{q})$ of an MEH-PPV:PCBM cell in a $1: 2$ weight ratio. 
blend of a bulk-heterojunction solar cell might have a different dark ideality factor. Note that in such a bulk-heterojunction solar cell, recombination only occurs at the donor-acceptor interface, which is a different situation than for double-carrier devices comprising a single organic semiconductor, such as OLEDs, in which recombination may occur everywhere in the bulk of the semiconductor.

To investigate whether the dark ideality factor of the solar cell is affected by the ideality factors of its components, we compared the $\eta-V$ characteristics of an MEH-PPV (poly[2-methoxy-5-(2-ethylhexyloxy)-1,4-phenylenevinylene]):PCBM solar cell and single-carrier diodes of its two constituents. As observed in Figure 11, the ideality factor of the dark current of the solar cells matches the ideality factor of a PCBM electron-only diode. This result is consistent with the fact that the electron mobility in PCBM is an order of magnitude higher than the hole mobility in MEH-PPV; thus, the dark current of the solar cell is dominated by the electron transport through the PCBM phase. ${ }^{41}$ For an MEH-PPV hole-only diode, an ideality factor of 1.6 was measured, which is consistent with the hole-only diode of the MEH-PPV:PCBM blend. It should be noted that in these singlecarrier diodes recombination is completely absent. It can therefore be concluded that the dark ideality factor of the blend is determined by the dominant type of charge carrier, resulting in a PCBM ideality factor of 1.3. An important prerequisite for dominant electron transport is that percolative pathways in the fullerene network exist such that charge transport is not limited by the microstructure of the blend. We therefore validated that an ideality factor of 1.3 was also measured in solar cells with a lower fullerene content, such as $\mathrm{MEH}$ PPV:PCBM in a 1:2 ratio and poly(3-hexylthiophene):PCBM in a 1:1 ratio. The occurrence of the fullerene ideality factor in a solar cell is also expected for other fullerene derivatives that exhibit virtually trap-free electron transport such that the dominant recombination mechanism is bimolecular. Indeed, for the $\mathrm{C}_{70}$ analogue of PCBM we also observed similar differences between the ideality factor in dark and under illumination.

In general, $V_{\mathrm{oc}}$ is determined by a competition between the recombination and generation of charges. ${ }^{34}$ Therefore, recombination mechanisms can be excellently extracted by studying the open-circuit voltage as a function of the generation rate, which can be

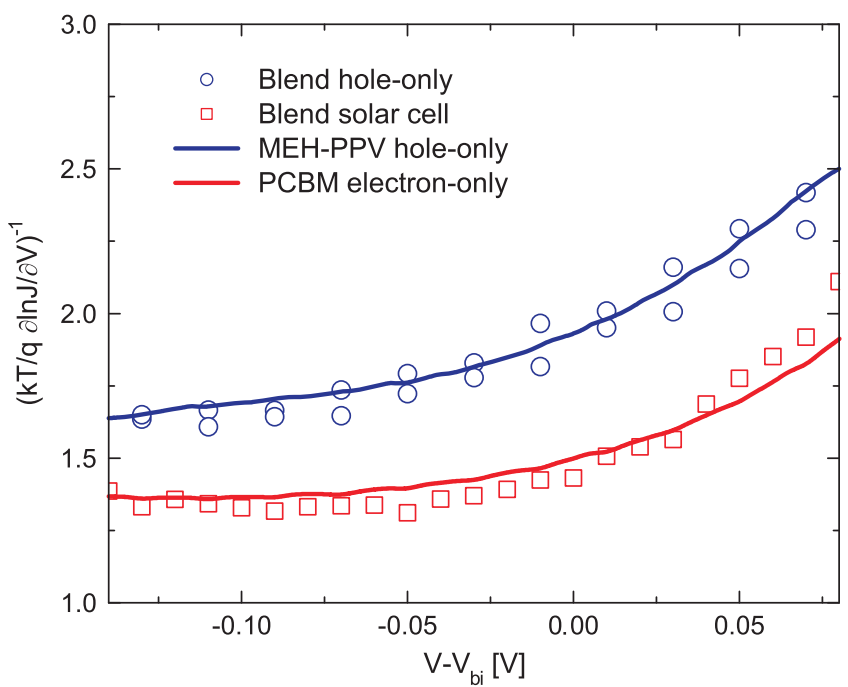

Figure $11 \eta-V$ characteristics of the dark current of an MEH-PPV:PCBM blend solar cell and hole-only device. The lines represent experimental $\eta-V$ characteristics for a MEH-PPV hole-only device and a PCBM electron-only device. tuned linearly by variation of the incident light intensity. For the solar cells investigated here, the dominant recombination mechanism is bimolecular, as evidenced by the slope of $k T / q$ in the $V_{\text {oc }}$-intensity measurements in Figure 10. Apparently, the light-intensity dependence of $V_{\mathrm{oc}}$ is not affected by the individual ideality factors of the donor and acceptor materials in the blend. The reason is that the deeply trapped carriers that cause greater-than-unity ideality factors in the dark current of the separate materials are released from the trap by recombination with photogenerated charges, such that their effect on the ideality factor is neutralized. Thus, the light-intensity dependence of $V_{\mathrm{oc}}$ can be used as a reliable tool to evaluate the presence of trap-assisted recombination.

A remaining issue is the large apparent temperature dependence of the ideality factor in organic solar cells, as observed in previous publications. $^{29,42}$ In section 3, we discussed a temperatureindependent ideality factor for single-carrier devices. If the dark current of the blend has an ideality factor equal to that of PCBM, the blend ideality factor should also be temperature-independent. This temperature independence can be directly observed for the MEH-PPV:PCBM cell in Figure 12. Therefore, these measurements confirm once more that the solar cell dark current and its ideality factor are dominated by the electron current through PCBM. Previous observations of a temperature-dependent ideality are likely caused by a too large leakage current. ${ }^{31,33}$

To conclude, this section has demonstrated that a deviation of the ideality factor from unity in the dark current of an organic bulkheterojunction solar cell cannot be used as evidence for the presence of trap-assisted recombination. As in single-carrier diodes, the ideality factor can deviate from unity even in the absence of recombination. It is shown that bimolecular recombination is dominant in these solar cells and that the non-ideality of the dark current is determined by the transport-dominating constituent in the donor:acceptor blend. ${ }^{43}$

\section{IDENTIFYING THE NATURE OF RECOMBINATION FROM CHARGE-TRANSFER STATE ELECTROLUMINESCENCE}

In section 2, we demonstrated for OLEDs that the recombination mechanism, either bimolecular or trap-assisted recombination, is directly reflected in the ideality factor of the current and light output. $^{33}$ However, the dark-current ideality factor of a polymer:fullerene bulk-heterojunction solar cell cannot give

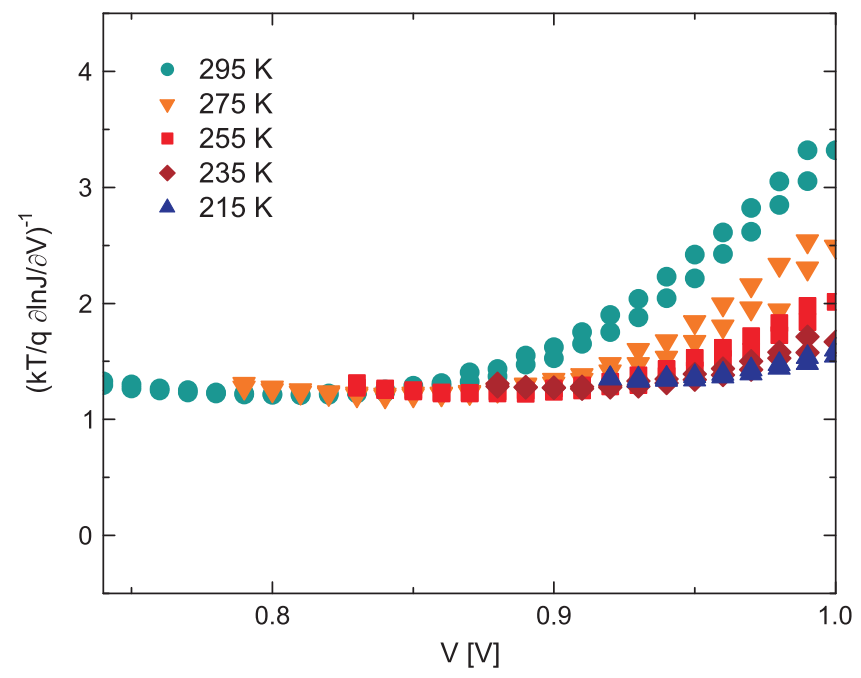

Figure 12 Temperature resolved $\eta-V$ characteristics of the dark current of an MEH-PPV:PCBM solar cell. 
conclusive information about the presence or absence of trap-assisted recombination. As described in a recent study by Tvingstedt et al., ${ }^{44}$ recombination at the polymer-fullerene interface is directly observable from the EL of the charge-transfer state. Charge-transfer state EL can be detected by applying a forward voltage across the solar cell and measuring the resulting emission with a silicon photodiode. Thus, the solar cell is simply operated as a light-emitting diode. As stated above, the different recombination mechanisms can directly be identified by measuring the luminance $(L)$ ideality factor, that is, the ideality factor of the EL intensity versus voltage characteristics. For bimolecular recombination, a luminance ideality factor of unity is expected, whereas radiative trap-assisted recombination is characterized by a luminance ideality factor of 2 .

In Figure 13, the total EL of the investigated bulk-heterojunction solar cells is plotted as a function of voltage. For all solar cells, weak luminescence could be detected, which is consistent with previous studies. ${ }^{44,45}$ At the onset of EL, the emission exhibits an exponential dependence on voltage according to $L \propto \exp (q V / \eta k T)$, similar to Equation (2). For the poly[3-hexylthiophene]:PCBM system, the EL is too weak to resolve the exponential part of the characteristics. At higher voltages, the emission bends off from the exponential, indicating that the injected current has reached the space-charge limit. The slope of the exponential luminescence $L$ is determined by the ideality factor, which, similar to Equation (3) for the current, can be directly obtained by numerical differentiation according to the following equation:

$$
\eta=\left(\frac{k T}{q} \frac{\partial \ln L}{\partial V}\right)^{-1}
$$

This derivative is plotted as a function of voltage in Figure 14, where the ideality factor can be obtained from the minimum or plateau value of the characteristics. ${ }^{33}$ Note that it is difficult to obtain a long plateau value because of weak EL and a limited sensitivity of the photodiode. It is clear from Figure 14 that the ideality factor approaches unity for the investigated solar cells. The ideality factor of 1 reveals that charge-transfer state emission originates from a free carrier, bimolecular recombination process. From these measurements, we can thus exclude the presence of radiative trap-assisted

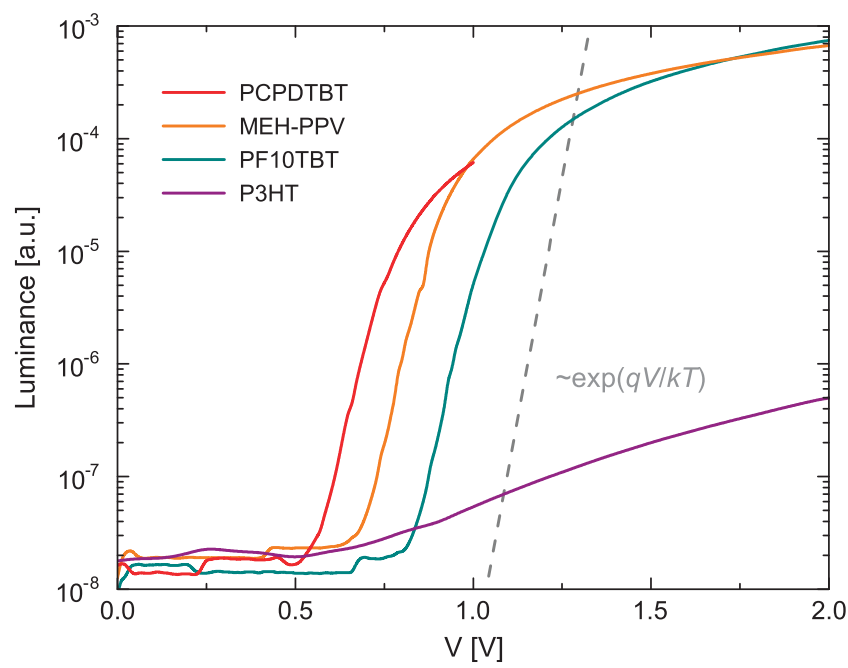

Figure 13 Luminance versus voltage characteristics for the charge-transfer state electroluminescence of several polymer:PCBM solar cells. The onset of emission is proportional to $\exp (q V / k T)$, plotted as a dashed line as a guide to the eye. recombination. ${ }^{46}$ This finding is consistent with the measurements on very similar systems by Tvingstedt et al., ${ }^{44}$ where no additional low-energy features could be observed in the CT state EL spectra.

\section{ANALYTICAL MODEL FOR DIFFUSION-LIMITED CURRENT IN ORGANIC MIM DIODES}

In section 3, it was demonstrated that in organic MIM diodes, the ideality factor deviates from unity because of the presence of energetically deep states within the band gap. However, a remarkable result was that even for organic semiconductors such as poly (9,9-dioctylfluorene) (PFO), the symmetrically substituted poly( $p$ phenylene vinylene) (PPV) derivative $\mathrm{BEH} / \mathrm{BB}-\mathrm{PPV}$, and the fullerene derivative PCBM, which exhibit nondispersive transport, ${ }^{47-49}$ pointing to the absence of deep traps, an ideality factor of typically 1.2-1.3 was observed instead of unity. To clarify this issue, an analytical equation that describes the diffusion current for undoped semiconductors or insulators in MIM diodes with asymmetric contacts must be derived.

Therefore, we use the diffusion theory by Schottky ${ }^{50}$ as a starting point. The original Schottky diffusion theory ${ }^{50}$ describes the situation for a metal contact on a doped semiconductor. In such a Schottky diode, majority of the carriers diffuse from the semiconductor into the metal to equilibrate the Fermi level. The charge of the remaining uncompensated dopants then leads to the build-up of an electric field in the depletion region, resulting in band bending. A similar model was used by Shockley for the current across a $p-n$ junction, viz a depletion region formed between a $p$ - and an $n$-doped semiconductor. ${ }^{3}$ An alternative theory to describe the current in a Schottky diode, based on thermionic emission, is valid only for high charge-carrier mobilities, ${ }^{51,52}$ whereas diffusion is the limiting case in low-mobility semiconductors, such as organic semiconductors.

In MIM structures, band bending due to uncompensated dopants is absent. Therefore, we will introduce the appropriate boundary conditions below. ${ }^{7}$ We start with the hole-only MIM diode of Figure 1a. Following the classical derivation of Schottky for diffusion currents, the hole current density is given as follows:

$$
J_{p}=\mu_{p} k T\left(\frac{p}{k T} \frac{\mathrm{d} E_{v}}{\mathrm{~d} x}-\frac{\mathrm{d} p}{\mathrm{~d} x}\right) \text {, }
$$

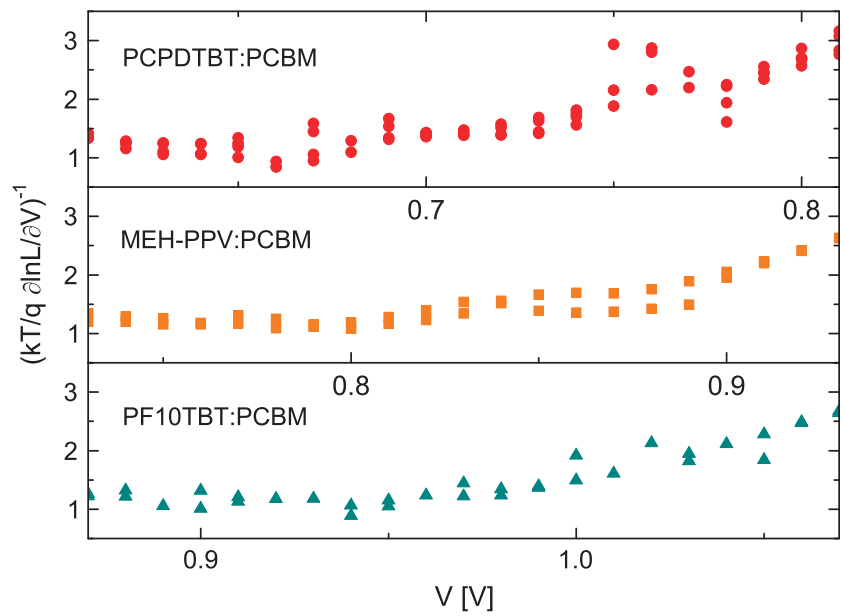

Figure 14 Luminance ideality factor of the charge-transfer state electroluminescence of the investigated polymer:PCBM solar cells obtained by numerical differentiation according to Equation (9). For all three systems, the curves approach unity at low voltages, which is indicative of a luminance ideality factor of 1 . 
where the diffusion coefficient has been replaced by the mobility $\mu_{p}$ through the Einstein relation. ${ }^{31}$ However, in contrast to a Schottky diode, the limit for integration in the MIM structure must be set to the total device thickness $L$ because the device is fully depleted, leading to:

$$
J_{p} \int_{0}^{L} \exp \left(-\frac{E_{v}}{k T}\right) \mathrm{d} x=-\left.\mu_{p} k T p(x) \exp \left(-\frac{E_{v}(x)}{k T}\right)\right|_{0} ^{L},
$$

where $p$ is the hole density and $E_{v}$ is the valence band edge or, analogously in this case, the HOMO of the polymer. To evaluate this expression, we must introduce the MIM boundary conditions for the charge-carrier density $p(x)$ and the valence band $E_{v}(x)$ at the electrode-semiconductor interfaces $x=0$ and $x=L$.

As illustrated in Figure $1 \mathrm{a}$, the $V_{\mathrm{bi}}$ for this device is equal to $\varphi_{\mathrm{b}}$. In that particular case, the boundary conditions for the charge-carrier density at the electrodes of a MIM device are given as follows ${ }^{7}$ :

$$
\begin{aligned}
& p(0)=N_{v}, \\
& p(L)=N_{v} \exp \left(-\frac{q \varphi_{\mathrm{b}}}{k T}\right),
\end{aligned}
$$

whereas the boundary conditions for the valence band, with respect to the Fermi level of the hole-extraction contact, for $V<V_{\mathrm{bi}}$ are given as follows:

$$
\begin{aligned}
& E_{v}(0)=-q V, \\
& E_{v}(L)=-q \varphi_{\mathrm{b}} .
\end{aligned}
$$

Note that in this case, a positive forward bias $V$ at $x=0$ reduces the internal voltage to $\varphi_{\mathrm{b}}-V$, leading to a reduction of the negative drift current of holes towards the injecting contact at $x=0$, which results in an enhanced positive current in the $x$ direction. Combination with Equation (11) yields the following:

$$
J_{p}=N_{v} \mu_{p} k T\left[\exp \left(\frac{q V}{k T}\right)-1\right] / \int_{0}^{L} \exp \left(-\frac{E_{v}(x)}{k T}\right) \mathrm{d} x .
$$

Because of the absence of space charge, there is no band bending in the MIM structure; therefore, the positional dependence of the valence band $E_{v}(x)$ is simply a triangular shape given as follows:

$$
E_{v}(x)=-q\left(V+\frac{\left(\varphi_{\mathrm{b}}-V\right) x}{L}\right),
$$

yielding the equation:

$$
\int_{0}^{L} \exp \left(-\frac{E_{v}(x)}{k T}\right) \mathrm{d} x=\frac{L k T}{q\left(\varphi_{\mathrm{b}}-V\right)}\left[\exp \left(\frac{q \varphi_{\mathrm{b}}}{k T}\right)-\exp \left(\frac{q V}{k T}\right)\right] .
$$

Substituting Equation (16) into Equation (14) then leads to an analytical current density expression for the diffusion-limited current in a MIM diode, given as follows:

$$
J_{p}=\frac{q \mu_{p} N_{v}\left(\varphi_{\mathrm{b}}-V\right)\left[\exp \left(\frac{q V}{k T}\right)-1\right]}{L\left[\exp \left(\frac{q \varphi_{\mathrm{b}}}{k T}\right)-\exp \left(\frac{q V}{k T}\right)\right]} .
$$

A similar expression was obtained by Paasch and coworkers ${ }^{53}$ We note that the use of a constant mobility in our derivation is valid because in the diffusion regime, the carrier densities in the diode are sufficiently low such that mobility enhancement due to DOS filling ${ }^{16}$ does not have a role. ${ }^{26,54}$ Before comparing Equation (17) to experimental data, it is essential to consider that for ohmic contacts on insulators or undoped semiconductors charge carriers diffuse from the electrode into the semiconductor, forming an accumulation region close to the contact. ${ }^{55}$ As illustrated in Figure 1b, the accumulated charge carriers close to the injecting contact at $x=0$ cause band bending and reduce the $V_{\mathrm{bi}}$ of the device. The reported values for this band bending, here described by the parameter $b$, typically lie in the range of $0.2-0.3 \mathrm{~V}$ and also depend on energetic disorder. ${ }^{56-60}$ To account for this accumulation, we approximate the energy-band diagram of the MIM diode by the dashed lines in Figure $1 \mathrm{~b}$. In this manner, the reduction of the $V_{\mathrm{bi}}$ is considered while maintaining the triangular potential. Then, the boundary conditions are modified as follows:

$$
\begin{aligned}
& p(0)=N_{v} \exp \left(-\frac{q b}{k T}\right), \\
& p(L)=N_{v} \exp \left(-\frac{q \varphi_{\mathrm{b}}}{k T}\right), \\
& E_{v}(0)=-q(V+b), \\
& E_{v}(L)=-q \varphi_{b} .
\end{aligned}
$$

The current equation, Equation (17), is for this case modified as follows:

$$
J_{p}=\frac{q \mu_{p} N_{v}\left(\varphi_{\mathrm{b}}-b-V\right)\left[\exp \left(\frac{q V}{k T}\right)-1\right]}{L \exp \left(\frac{q b}{k T}\right)\left[\exp \left(\frac{q\left(\varphi_{\mathrm{b}}-b\right)}{k T}\right)-\exp \left(\frac{q V}{k T}\right)\right]} .
$$

Clearly, the band-bending parameter $b$ does not only reduce the $V_{\mathrm{bi}}$ but also has a large effect on the current close to $V_{\mathrm{bi}}$ and above $V_{\mathrm{bi}}$. Therefore, we introduce the band-bending parameter $b$ as follows:

$$
b=\frac{k T}{q}\left[\ln \left(\frac{q^{2} N_{v} L^{2}}{2 k T \varepsilon}\right)-2\right],
$$

based on a model by Simmons. ${ }^{56,61}$ At $V_{\mathrm{bi}}$, the current undergoes a transition from an exponential to a linear dependence on voltage. Because of the thickness dependence of the band-bending parameter, the linear current above $V_{\mathrm{bi}}$ depends inversely on $L^{3}$, whereas the exponential part below $V_{\text {bi }}$ scales inversely with $L$. For voltages larger than the built-in voltage, the electric field becomes positive and the drift current starts to dominate. This drift current is the well-known space-charge-limited current, described by Equation (1). With the derived diffusion current, Equation (19), we can now obtain the complete current through the device by summing the contributions of drift and diffusion, as shown in Figure 15. The drift current starts precisely at the built-in voltage, well defined by $\varphi_{\mathrm{b}}-b$. The analytical expression for the diffusion current now allows disentanglement of the drift and diffusion contributions to the current.

When comparing the expression for the diffusion-limited current for MIM diodes, Equation (19), to the classical Shockley diode equation, Equation (2), it is clear that there are subtle differences in the voltage dependence of the current density, as shown in the inset of Figure 15. To match the slope of Equation (19) using an effective barrier, $\varphi_{\mathrm{b}}-b$, of $0.3 \mathrm{~V}$, the ideality factor in Equation (2) must be adjusted to 1.2. This observation explains why experimentally in organic semiconductors without deep traps, small deviations from unity of the ideality factor of $\sim 0.2$ were observed when the Shockley equation was used for the analysis. This slight deviation from unity is therefore a fundamental property of organic MIM diodes and is related to the charge transport. We note that the exact value of the deviation depends on the value of the effective applied voltage, $\varphi_{\mathrm{b}}-b-V$. For most organic MIM diodes, the ideality factor is determined within $0.3 \mathrm{~V}$ below the $V_{\mathrm{bi}}\left(\varphi_{\mathrm{b}}-b\right),{ }^{31}$ leading to an apparent ideality factor of $\sim 1.2$. For large barriers, carriers of the 


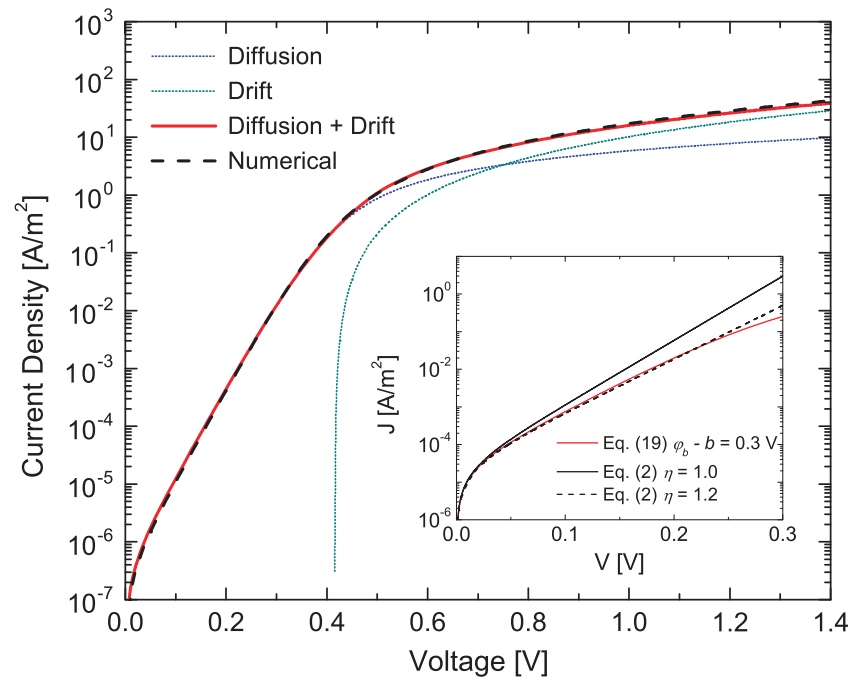

Figure 15 Current density-voltage characteristics for a device with $\varphi_{\mathrm{b}}=0.7 \mathrm{~V}, \quad \mu_{p}=1 \times 10^{-9} \mathrm{~m}^{2} \mathrm{Vs}^{-1}, \quad N_{v}=3 \times 10^{26} \mathrm{~m}^{-3}, \quad L=100 \mathrm{~nm}$, $T=295 \mathrm{~K}$ and $\varepsilon=3 \varepsilon_{0}$. The dotted and solid lines are the analytically calculated characteristics for diffusion, drift and the sum of drift and diffusion. The dashed line represents a numerical simulation ${ }^{7}$ with the exactly same parameters. The inset shows a comparison between the calculated current for a MIM diode, Equation (19), with $\varphi_{\mathrm{b}}-b=0.3 \mathrm{~V}$ (red line) and the current calculated with the classical Shockley equation using an ideality factor of unity (solid black line). A good approximation is obtained using an ideality factor of 1.2 (dashed line).

opposite sign will be injected, leading to trap-assisted recombination that will enhance the ideality factor. ${ }^{33}$

To evaluate the applicability of the expression derived for the diffusion-limited current in MIM diodes, we investigated a PFO holeonly diode with PEDOT:PSS and $\mathrm{MoO}_{3}$ contacts. $\mathrm{MoO}_{3}$ provides an ohmic contact even to PFO with a $\mathrm{HOMO}$ as deep as $5.8 \mathrm{eV}^{62}$ The work function of PEDOT:PSS is $\sim 5.2 \mathrm{eV}$. Hence, PEDOT:PSS serves as the collecting contact with an estimated energy barrier of $\sim 0.6 \mathrm{eV}$. The exact value is not a priori known and may depend on the presence of interface dipoles and on Fermi level pinning because of interface trap states.

The experimental temperature-dependent current-voltage characteristics are presented in Figure 16. At low bias, the current increases exponentially with bias as expected for a diffusion-limited current. Above $\sim 0.4-0.5 \mathrm{~V}$, the current becomes drift-dominated and is limited by the uncompensated charges of the injected holes, leading to a space-charge-limited current.

The parameters describing the charge-carrier mobility of PFO as a function of carrier density and temperature are well known. ${ }^{62}$ The diffusion-limited current can now be fitted to the experimental data by adjusting the barrier in Equation (19). The solid lines are a fit to the experimental data. Good agreement in the diffusion regime at $V<V_{\mathrm{bi}}$ is obtained using a barrier $\varphi_{\mathrm{b}}$ of $0.67 \mathrm{~V}$, with a band-bending parameter of $0.26 \mathrm{~V}$ (Equation (20)). The value for the barrier corresponds to previous estimates of the energy barrier between PFO and PEDOT:PSS of $\sim 0.6 \mathrm{eV}^{62}$

The dashed lines in Figure 16 represent numerical device calculations using a drift-diffusion solver, ${ }^{7}$ with precisely the same parameters as used in the analytical fits. The numerical simulation contains both drift and diffusion, and band-bending at the injecting contact is implicitly considered. The good agreement verifies the analytical expression of the diffusion-limited current in an organic MIM diode. The deviations for $V>V_{\mathrm{bi}}$ are because of the current

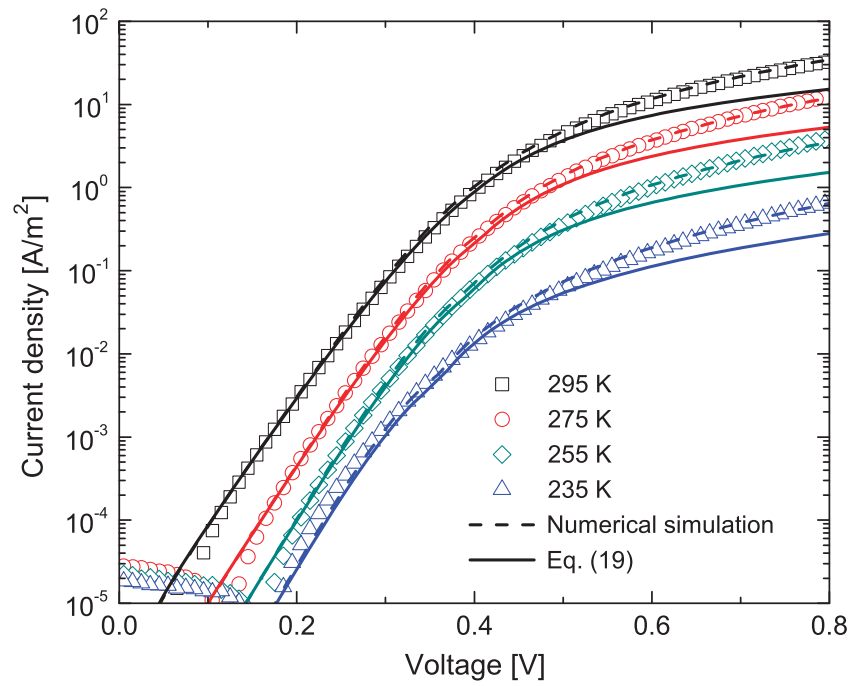

Figure 16 Current-voltage characteristics of a PEDOT:PSS/PFO $(80 \mathrm{~nm}) /$ $\mathrm{MoO}_{3}$ hole-only MIM diode. PEDOT:PSS is grounded. The solid lines are fits to the experimental data (symbols) using the analytical equation for the diffusion-limited current. The deviation at higher voltages is due to the current being dominated by the drift term (space-charge-limited current, Equation (1)) above the built-in voltage. The dashed lines represent numerical device calculations using a drift-diffusion solver ${ }^{7}$ with exactly the same parameters as extracted from the analytical description.

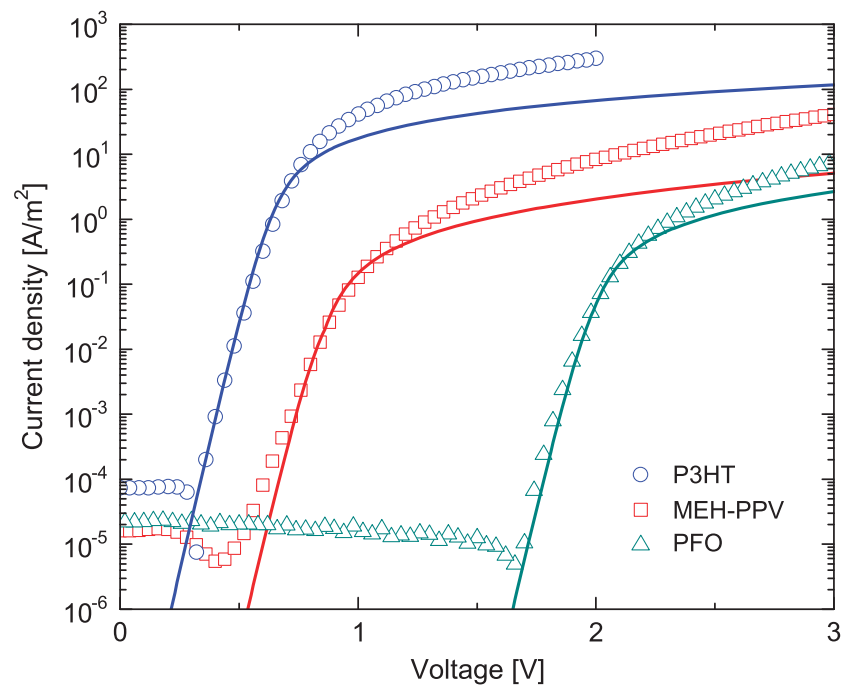

Figure 17 Experimental current-voltage characteristics (symbols) of organic $\mathrm{MIM}$ diodes with an Ohmic $\mathrm{MoO}_{3}$ contact and a $\mathrm{ZnO}$ collecting contact. The poly[3-hexylthiophene], MEH-PPV and PFO layer thicknesses are 125, 120 and $180 \mathrm{~nm}$, respectively. The solid lines are fits with Equation (19).

being dominated by the drift term at those voltages. With the $b$ parameter known, the analytical description for the diffusion-limited current in MIM diodes can be used to accurately determine the $V_{\mathrm{bi}}$ and injection barrier of the collecting contact. As an example, we investigated hole-only diodes of the polymers poly[3-hexylthiophene], MEH-PPV and PFO with oxidic electrodes. $\mathrm{MoO}_{3}$ was used as an ohmic hole-injecting contact. $\mathrm{ZnO}$ was used as the collecting contact. The work function of $\mathrm{ZnO}$ is $\sim 4.0 \mathrm{eV}$. Considering the reported HOMO energies of $-4.8,-5.1$ and $-5.8 \mathrm{eV}$, expected energy barriers of $0.8,1.1$ and $1.8 \mathrm{eV}$ are obtained for poly[3-hexylthiophene], MEH-PPV and PFO, respectively. 
The current-voltage characteristics are presented in Figure 17. At very low bias, the current is below the detection limit of the measurement set-up. With increasing bias, the exponential diffusion current exceeds the noise level. The solid lines represent fits of the diffusion-limited current in MIM diodes to the experimental data. The values for the barriers extracted are $0.93,1.15$ and $2.35 \mathrm{~V}$, respectively. The obtained barriers agree well with the estimated energy barriers. This observation confirms the previous findings that the electron chemical potential is the main driving force for energylevel alignment at contacts consisting of an organic semiconductor and transition-metal oxides. ${ }^{63}$

In summary, this section described the derivation of an analytical equation for the diffusion current in a MIM diode. Summing with the drift current results in a full analytical description of the current. It was demonstrated that a slight deviation of the apparent ideality factor from unity in single-carrier devices is characteristic of organic MIM diodes. Furthermore, the equation for the diffusion current can be used to determine the $V_{\mathrm{bi}}$ and injection barrier in organic diodes.

\section{CONCLUSIONS}

In this review, it was demonstrated that the ideality factor of diffusion-driven currents can be regarded as an effective tool for studying the recombination mechanisms in OLEDs and solar cells. In OLEDs, the ideality factor has a value of 2 because of nonradiative trap-assisted recombination. By contrast, the light output of such a device has an ideality factor approaching unity, indicating that the luminescence is governed by bimolecular free-carrier recombination. Whereas an ideality factor above unity can be regarded as a sign of trap-assisted recombination, an increased ideality factor can even be measured for single-carrier devices, in which no recombination occurs. The non-ideality $(\eta>1)$ is caused by a small number of deeply trapped carriers, of which the effect is neutralized by chargecarrier recombination. Similarly, in organic solar cells, the ideality factor of the dark current is dominated by the fastest transporting material in the blend and therefore cannot be used as exclusive evidence for the presence or absence of trap-assisted recombination. Finally, an analytical approach to model the diffusion current in organic MIM diodes with one ohmic and one non-ohmic contact was developed. The analytical model accurately describes the voltage, temperature and thickness dependence of the diffusion current and thus allows disentanglement of the drift and diffusion contributions to the current. It was demonstrated that a slight deviation of the apparent ideality factor from unity in single-carrier devices is characteristic of organic MIM diodes. Applying the analytical equation to experimental data provides an easy and accurate method of determining the $V_{\mathrm{bi}}$ and injection barrier in organic MIM diodes.

\section{ACKNOWLEDGEMENTS}

We gratefully acknowledge the contributions of LJA Koster, M Kuik, HT Nicolai, M Lenes, P de Bruyn, DM de Leeuw and J Harkema. The work of GAHW formed part of the research program of the Dutch Polymer Institute, Project No. 678

1 Mott, N. F. \& Gurney, R. W. Electronic Processes in Ionic Crystals (Oxford University Press, London, 1940).

2 Blom, P. W. M., de Jong, M. J. M. \& Vleggaar, J. J. M. Electron and hole transport in poly(p-phenylene vinylene) devices. Appl. Phys. Lett. 68, 3308-3310 (1996).

3 Shockley, W. The theory of $p-n$ junctions in semiconductors and $p-n$ junction transistors. Bell Syst. Tech. J. 28, 435-489 (1949).

4 Sah, C. T., Noyce, R. N. \& Shockley, W. Carrier generation and recombination in P-N junctions and P-N junction characteristics. Proc. IRE 45, 1228-1243 (1957).
5 Shockley, W. \& Read, W. T. Statistics of the recombinations of holes and electrons. Phys. Rev. 87, 835-842 (1952).

6 Hall, R. N. Electron-hole recombination in germanium. Phys. Rev. 87, 387 (1952).

7 Koster, L. J. A., Smits, E. C. P., Mihailetchi, V. D. \& Blom, P. W. M. Device model for the operation of polymer/fullerene bulk heterojunction solar cells. Phys. Rev. B 72 , 085205 (2005)

8 Blom, P. W. M., de Jong, M. J. M. \& Breedijk, S. Temperature dependent electron-hole recombination in polymer light-emitting diodes. Appl. Phys. Lett. 71, 930-932 (1997).

9 Pivrikas, A., Juška, G., Österbacka, R., Westerling, M., Viliūnas, M., Arlauska, K. \& Stubb, H. Langevin recombination and space-charge-perturbed current transients in regiorandom poly(3-hexylthiophene). Phys. Rev. B 71, 125205 (2005).

10 Langevin, P. Recombinaison et mobilités des ions dans les gaz. Ann. Chim. Phys. 28, 433-530 (1903).

11 De Kok, M., Sarfert, W. \& Paetzold, R. Tuning the colour of white polymer light-emitting diodes. Thin Solid Films 518, 5265-5271 (2010).

12 Parshin, M. A., Ollevier, J., Van der Auweraer, M., De Kok, M. M., Nicolai, H. T. Hof, A. J. \& Blom, P. W. M. Hole transport in blue and white emitting polymers. J. Appl. Phys. 103, 113711 (2008).

13 Einstein, A. Über die von der molekularkinetischen Theorie der Wärme geforderte Bewegung von in ruhenden Flüssigkeiten suspendierten Teilchen. Ann. Phys. 322, 549-560 (1905)

14 Bässler, H. Charge transport in disordered organic photoconductors a Monte Carlo simulation study. Phys. Status Solidi B 175, 15-56 (1993).

15 Novikov, S. V., Dunlap, D. H., Kenkre, V. M., Parris, P. E. \& Vannikov, A. V. Essentia role of correlations in governing charge transport in disordered organic materials. Phys. Rev. Lett. 81, 4472-4475 (1998).

16 Vissenberg, M. C. J. M. \& Matters, M. Theory of the field-effect mobility in amorphous organic transistors. Phys. Rev. B 57, 12964-12967 (1998)

17 Richert, R., Pautmeier, L. \& Bässler, H. Diffusion and drift of charge carriers in random potential: deviation from Einstein's law. Phys. Rev. Lett. 63, 547-550 (1989).

18 Borsenberger, P. M., Pautmeier, L., Richert, R. \& Bässler, H. Hole transport in 1,1-bis(di-4-tolylaminophenyl)cyclohexane. J. Chem. Phys. 94, 8276-8281 (1991).

19 Baranovskii, S. D. Faber, T., Hensel, F., Thomas, P. \& Adriaenssens, G. J. Einstein's relationship for hopping electrons. J. Non-Cryst. Solids 198-200, 214-217 (1996).

20 Nenashev, A. V., Jansson, F., Baranovskii, S. D. Österbacka, R., Dvurechenskii, A. V. \& Gebhard, F. Effect of electric field on diffusion in disordered materials. II. Two- and three-dimensional hopping transport. Phys. Rev. B 81, 115204 (2010).

21 Ritter, D., Zeldov, E. \& Weiser, K. Ambipolar transport in amorphous semiconductors in the lifetime and relaxation-time regimes investigated by the steady-state photocarrie grating technique. Phys. Rev. B 38, 8296-8304 (1988).

22 Roichman, Y. \& Tessler, N. Generalized Einstein relation for disordered semiconductors - implications for device performance. Appl. Phys. Lett. 80, 1948-1950 (2002).

23 Harada, K., Werner, A. G., Pfeiffer, M., Bloom, C. J., Elliott, C. M. \& Leo, K. Organic homojunction diodes with a high built-in potential: interpretation of the current-voltage characteristics by a generalized Einstein relation. Phys. Rev. Lett. 94, 036601 (2005).

24 Baranovskii, S. D., Faber, T., Hensel, F. \& Thomas, P. On the Einstein relation for hopping electrons. J. Non-Cryst. Solids 227-230, 158-161 (1998).

25 Tal, O., Epstein, I., Snir, O., Roichman, Y., Ganot, Y., Chan, C. K., Kahn, A., Tessler, N. \& Rosenwaks, $Y$. Measurements of the Einstein relation in doped and undoped molecular thin films. Phys. Rev. B 77, 201201 (2008)

26 Tanase, C., Meijer, E. J., Blom, P. W. M. \& De Leeuw, D. M. Unification of the hole transport in polymeric field-effect transistors and light-emitting diodes. Phys. Rev. Lett. 91, 216601 (2003).

27 Tessler, N. \& Roichman, Y. Amorphous organic molecule/polymer diodes and transistors - comparison between predictions based on Gaussian or exponential density of states. Org. Electron. 6, 200-210 (2005).

28 Vaynzof, Y., Preezant, Y. \& Tessler, N. Current voltage relation of amorphous materials based $\mathrm{pn}$ diodes - the effect of degeneracy in organic polymers/molecules. J. Appl. Phys. 106, 084503 (2009).

29 Koster, L. J. A., Mihailetchi, V. D., Ramaker, R. \& Blom, P. W. M. Light intensity dependence of open-circuit voltage of polymer:fullerene solar cells. Appl. Phys. Lett. 86, 123509 (2005)

30 Harada, K., Riede, M., Leo, K., Hild, O. R. \& Elliott, C. M. Pentacene homojunctions: electron and hole transport properties and related photovoltaic responses. Phys. Rev. B 77, 195212 (2008)

31 Wetzelaer, G. A. H., Koster, L. J. A. \& Blom, P. W. M. Validity of the Einstein relation in disordered organic semiconductors. Phys. Rev. Lett. 107, 066605 (2011).

32 Craciun, N. I., Wildeman, J. \& Blom, P. W. M. Universal Arrhenius temperature activated charge transport in diodes from disordered organic semiconductors. Phys. Rev. Lett. 100, 056601 (2008).

33 Wetzelaer, G. A. H., Kuik, M., Nicolai, H. T. \& Blom, P. W. M. Trap-assisted and Langevin-type recombination in organic light-emitting diodes. Phys. Rev. B 83, 165204 (2011).

34 Maurano, A., Hamilton, R., Shuttle, C. G., Ballantyne, A. M., Nelson, J., O'Regan, B., Zhang, W., McCulloch, I., Azimi, H., Morana, M., Brabec, C. J. \& Durrant, J. R. Recombination dynamics as a key determinant of open circuit voltage in organic bulk heterojunction solar cells: a comparison of four different donor polymers. Adv. Mater. 22, 4987-4992 (2010).

35 Mihailetchi, V. D., van Duren, J. K. J., Blom, P. W. M., Hummelen, J. C., Janssen, R. A J., Kroon, J. M., Rispens, M. T., Verhees, W. J. H. \& Wienk, M. M. Electron transport in a methanofullerene. Adv. Funct. Mater. 13, 43-46 (2003). 
36 Street, R. A., Schoendorf, M., Roy, A. \& Lee, J. H. Interface state recombination in organic solar cells. Phys. Rev. B 81, 205307 (2010).

37 Kirchartz, T., Pieters, B. E., Kirkpatrick, J., Rau, U. \& Nelson, J. Recombination via tail states in polythiophene:fullerene solar cells. Phys. Rev. B 83, 115209 (2011).

38 Cowan, S. R., Leong, W. L., Banerji, N., Dennler, G. \& Heeger, A. J. Identifying a threshold impurity level for organic solar cells: enhanced first-order recombination via well-defined PC84BM traps in organic bulk heterojunction solar cells. Adv. Funct. Mater. 21, 3083-3092 (2011).

39 Mandoc, M. M., Kooistra, F. B., Hummelen, J. C., De Boer, B. \& Blom, P. W. M. Effect of traps on the performance of bulk heterojunction organic solar cells. Appl. Phys. Lett. 91, 263505 (2007)

40 Mandoc, M. M., Veurman, W., Koster, L. J. A., De Boer, B. \& Blom, P. W. M. Origin of the reduced fill factor and photocurrent in MDMO-PPV:PCNEPV all-polymer solar cells. Adv. Funct. Mater. 17, 2167-2173 (2007).

41 Blom, P. W. M., Mihailetchi, V. D., Koster, L. J. A. \& Markov, D. E. Device physics of polymer:fullerene bulk heterojunction solar cells. Adv. Mater. 19, 1551-1566 (2007).

42 Riedel, I., Von Hauff, E., Parisi, J., Martín, N., Giacalone, F. \& Dyakonov, V. Diphenylmethanofullerenes: new and efficient acceptors in bulk-heterojunction solar cells. Adv. Funct. Mater. 15, 1979-1987 (2005).

43 Wetzelaer, G. A. H., Kuik, M., Lenes, M. \& Blom, P. W. M. Origin of the dark-current ideality factor in polymer:fullerene bulk heterojunction solar cells. Appl. Phys. Lett. 99, 153506 (2011).

44 Tvingstedt, K., Vandewal, K., Gadisa, A., Zhang, F., Manca, J. \& Inganas, 0. Electroluminescence from charge transfer states in polymer solar cells. J. Am. Chem. Soc. 131, 11819-11824 (2009).

45 Vandewal, K., Tvingstedt, K., Gadisa, A., Inganäs, O. \& Manca, J. V. On the origin of the open-circuit voltage of polymer-fullerene solar cells. Nat. Mater. 8, 904-909 (2009).

46 Wetzelaer, G. A. H., Kuik, M. \& Blom, P. W. M. Identifying the nature of charge recombination in organic solar cells from charge-transfer state electroluminescence. Adv. Energy Mater. 2, 1232-1237 (2012).

47 Campbell, A. J., Bradley, D. D. C. \& Antoniadis, H. Quantifying the efficiency of electrodes for positive carrier injection into poly(9,9-dioctylfluorene) and representative copolymers. J. Appl. Phys. 89, 3343-3351 (2001).

48 Tanase, C., Blom, P. W. M., De Leeuw, D. M. \& Meijer, E. J. Charge carrier density dependence of the hole mobility in poly(p-phenylene vinylene). Phys. Status Solidi $A$ 201, 1236-1245 (2004).

49 Tuladhar, S. M., Poplavskyy, D., Choulis, S. A., Durrant, J. R., Bradley, D. D. C. \& Nelson, J. Ambipolar charge transport in films of methanofullerene and Poly(phenylenevinylene)/methanofullerene blends. Adv. Funct. Mater. 15, 1171-1182 (2005)

50 Schottky, W. Halbleitertheorie der Sperrschicht. Naturwissenschaften 26, 843 (1938).

51 Simmons, J. G. Richardson-Schottky effect in solids. Phys. Rev. Lett. 15, 967-968 (1965).
52 Sze, S. M. Physics of Semiconductor Devices (Wiley-Interscience, New York, NY, USA, 1981).

53 Nguyen, P. H., Scheinert, S., Berleb, S., Brütting, W. \& Paasch, G. The influence of deep traps on transient current-voltage characteristics of organic light-emitting diodes. Org. Electron. 2, 105-120 (2001).

54 Pasveer, W. F., Cottaar, J., Tanase, C., Coehoorn, R., Bobbert, P. A., Blom, P. W. M., De Leeuw, D. M. \& Michels, M. A. J. Unified description of charge-carrier mobilities in disordered semiconducting polymers. Phys. Rev. Lett. 94, 206601 (2005).

55 Craciun, N. I., Brondijk, J. J. \& Blom, P. W. M. Diffusion-enhanced hole transport in thin polymer light-emitting diodes. Phys. Rev. B 77, 035206 (2008).

56 Simmons, J. G. Theory of metallic contacts on high resistivity solids - I. Shallow traps. J. Phys. Chem. Solids 32, 1987-1999 (1971).

57 Mihailetchi, V. D., Blom, P. W. M., Hummelen, J. C. \& Rispens, M. T. Cathode dependence of the open-circuit voltage of polymer:fullerene bulk heterojunction solar cells. J. Appl. Phys. 94, 6849-6854 (2003).

58 Hwang, J., Kim, E. G., Liu, J., Bredas, J.-L., Duggal, A. \& Kahn, A. Photoelectron spectroscopic study of the electronic band structure of polyfluorene and fluorenearylamine copolymers at interfaces. J. Phys. Chem. C 111, 1378-1384 (2007).

59 Blakesley, J. C. \& Greenham, N. C. Charge transfer at polymer-electrode interfaces: The effect of energetic disorder and thermal injection on band bending and open-circuit voltage. J. Appl. Phys. 106, 034507 (2009).

60 Lange, I., Blakesley, J. C., Frisch, J., Vollmer, A., Koch, N. \& Neher, D. Band bending in conjugated polymer layers. Phys. Rev. Lett. 106, 216402 (2011).

61 De Bruyn, P., Van Rest, A. H. P., Wetzelaer, G. A. H., De Leeuw, D. M. \& Blom, P. W. M. Diffusion-limited current in organic metal-insulator-metal diodes. Phys. Rev. Lett. 111, 186801 (2013).

62 Nicolai, H. T., Wetzelaer, G. A. H., Kuik, M., Kronemeijer, A. J., De Boer, B. \& Blom, P. W. M. Space-charge-limited hole current in poly(9,9-dioctylfluorene) diodes. Appl. Phys. Lett. 96, 172107 (2010).

63 Greiner, M. T., Helander, M. G., Tang, W.-M., Wang, Z.-B., Qiu, J. \& Lu, Z.-H. Universal energy-level alignment of molecules on metal oxides. Nat. Mater. 11, 76-81 (2012).

(i) This work is licensed under a Creative Commons Attribution 3.0 Unported License. The images or other third party material in this article are included in the article's Creative Commons license, unless indicated otherwise in the credit line; if the material is not included under the Creative Commons license, users will need to obtain permission from the license holder to reproduce the material. To view a copy of this license, visit http:// creativecommons.org/licenses/by/3.0/
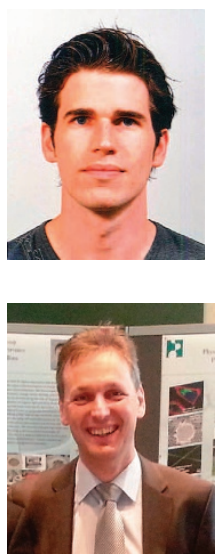

Gert-Jan Wetzelaer obtained an MSc degree in Applied Physics at the University of Groningen in 2009. In 2014, he obtained a PhD degree from the University of Groningen. His doctoral research focused on charge transport and recombination in organicsemiconductor diodes, under supervision of Professor Paul Blom. In particular, diffusion currents and related phenomena were investigated. Currently, he works as a postdoctoral researcher on biophotovoltaic cells involving photosynthetic proteins.
Paul WM Blom, born in 1965 in The Netherlands, received his PhD Degree in 1992 from the Technical University Eindhoven on picosecond charge carrier dynamics in GaAs. At Philips Research Laboratories he was engaged in the electro-optical properties of polymer light-emitting diodes. From 2000 he held a professorship at the University of Groningen in the field of electrical and optical properties of organic semiconducting devices. In September 2008 he became Scientific Director of the Holst Centre in Eindhoven, where the focus is on foil-based electronics, followed in 2012 by an appointment as director at the MPI for polymer research in the field of molecular electronics. 